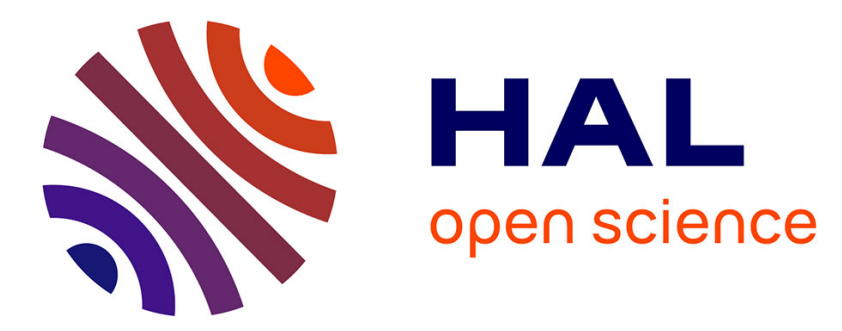

\title{
New characterizations for the eigenvalues of the prolate spheroidal wave equation
}

Françoise Richard-Jung, Jean-Pierre Ramis, Jean Thomann, Frédéric Fauvet

\section{To cite this version:}

Françoise Richard-Jung, Jean-Pierre Ramis, Jean Thomann, Frédéric Fauvet. New characterizations for the eigenvalues of the prolate spheroidal wave equation. Studies in Applied Mathematics, 2017, 138 (1), pp.3-42. 10.1111/sapm.12134 . hal-01151663

\section{HAL Id: hal-01151663 \\ https://hal.science/hal-01151663}

Submitted on 13 May 2015

HAL is a multi-disciplinary open access archive for the deposit and dissemination of scientific research documents, whether they are published or not. The documents may come from teaching and research institutions in France or abroad, or from public or private research centers.
L'archive ouverte pluridisciplinaire HAL, est destinée au dépôt et à la diffusion de documents scientifiques de niveau recherche, publiés ou non, émanant des établissements d'enseignement et de recherche français ou étrangers, des laboratoires publics ou privés. 


\title{
New characterizations for the eigenvalues of the prolate spheroidal wave equation
}

\author{
By F. Richard-Jung, J.-P. Ramis, J. Thomann, F. Fauvet
}

In this paper, we give new characterizations for the eigenvalues of the prolate wave equation as limits of the zeros of some families of polynomials: the coefficients of the formal power series appearing in the solutions near 0,1 or $\infty$ (in the variables $x, x-1$ or $1 / x$ respectively). The result, which seems to be true for all values of the parameter $\tau$, according to our numerical experiments, is here proved for small values of the parameter $\tau$.

\section{Introduction}

This paper is devoted to the study of the spectral problem $\mathcal{D}_{\tau} y=\mu y$ where:

$$
\mathcal{D}_{\tau}: y \mapsto\left(x^{2}-1\right) y^{\prime \prime}+2 x y^{\prime}+\tau^{2} x^{2} y,
$$

$\tau$ being a real parameter $(\tau \geq 0)$.

It is equivalent to the study of the spectral problem for the prolate spheroidal wave operators ${ }^{1}$ but $\mathcal{D}_{\tau}$, being a perturbation of the opposite of the Legendre operator:

$$
\mathcal{D}_{0}=\left(x^{2}-1\right)\left(\frac{d}{d x}\right)^{2}+2 x \frac{d}{d x}=-\frac{d}{d x}\left(\left(1-x^{2}\right) \frac{d}{d x}\right),
$$

is more adapted to a perturbative approach.

The differential operator $\mathcal{D}_{\tau}$ is interpreted as defined on the Riemann sphere $P_{1}(\mathbb{C}):=$ $\mathbb{C} \cup\{\infty\}$. It admits 3 singularities: $x=1, x=-1, x=\infty ; 1$ and -1 are regular-singular and $\infty$ is irregular. The differential equation $\mathcal{D}_{\tau} y-\mu y=0$ is a confluent Heun equation.

In a preceding paper [1], we made the following observations.

For $\tau \geq 0$ fixed and $\mu \in \mathbb{R}$, the following properties are equivalent:

1. $\mu$ is not an eigenvalue of $\mathcal{D}_{\tau}$;

Francoise.Jung@imag.fr, ramis@picard.ups-tlse.fr, thomann@math.u-strasbg.fr, fauvet@math.u-strasbg.fr ${ }^{1}$ Of order $m=0$. 
2. a non trivial power series solution of $\mathcal{D}_{\tau} y-\mu y=0$ at $x=1$ admits 2 as convergence radius;

3. a non trivial power series solution of $\mathcal{D}_{\tau} y-\mu y=0$ at $x=-1$ admits 2 as convergence radius;

4. the non trivial power series solution of $\mathcal{D}_{\tau} y-\mu y=0$ at $x=0$ admits 1 as convergence radius;

5. the power series solutions appearing in a fundamental system of formal solutions of $\mathcal{D}_{\tau} y-\mu y=0$ at $x=\infty$ are divergent.

For $\tau \geq 0$ fixed and $\mu \in \mathbb{R}$, the following properties are equivalent:

1. $\mu$ is an eigenvalue of $\mathcal{D}_{\tau}$;

2. a power series solution of $\mathcal{D}_{\tau} y-\mu y=0$ at $x=1$ admits $+\infty$ as convergence radius;

3. a power series solution of $\mathcal{D}_{\tau} y-\mu y=0$ at $x=-1$ admits $+\infty$ as convergence radius;

4. there exists a non trivial even or odd power series solution of $\mathcal{D}_{\tau} y-\mu y=0$ at $x=0$ admitting $+\infty$ as convergence radius;

5. the power series solutions of $\mathcal{D}_{\tau} y-\mu y=0$ appearing in the formal series solutions at $x=\infty$ are convergent.

In the above observations all the power series (respectively in the variables $x-1, x+1$, $x, 1 / x)$ admits as coefficients some polynomials in $\mu$ with coefficients in $\mathbb{C}\left[\tau, \tau^{-1}\right]$, satisfying some polynomial linear recurrences. For example there exists a unique even power series solution at $x=0: \sum_{n \in \mathbb{N}} P_{2 n} x^{2 n}, P_{0}:=1$. (The $P_{2 n}$ satisfy a three terms recurrence, they are polynomials of degree $n$ in the variable $\mu$ with coefficients in $\mathbb{Q}[\tau]$.)

For a fixed value of the parameter $\tau$ and $\mu \in \mathbb{R}$, the problem to decide if $\mu$ is an eigenvalue of $\mathcal{D}_{\tau}$ is a priori a global problem but it follows from the above observations that this problem can be "solved" locally at $x=1$, or $x=-1$, or $x=0$, or $x=\infty$. For example at $x=0$ we have to "see" if the radius of convergence of $\sum_{n \in \mathbb{N}} P_{2 n}(\mu) x^{2 n}$ is $+\infty$ or finite.

The source of our article is the following experimental observation. (We will explain what is happening at $x=0$ but there are similar phenomena for $x=1, x=-1$ or $x=\infty$.) Trying to use the above considerations, that is the "jump" in the radius of convergence when $\mu$ crosses an eigenvalue, to get an heuristic "quick and efficient" numerical method to compute the eigenvalues ${ }^{2}$, we discovered a surprising phenomenon: experimentally, for all $j \in \mathbb{N}$, the $j$-th zero of the polynomial $P_{2 p}(p \geq j)$ tends to the $2 j$-th eigenvalue of $\mathcal{D}_{\tau}$ when $p$ tends to $+\infty^{3}$.

Our article contains a proof of this result and of its variations at $x=1, x=-1$ or $x=\infty .{ }^{4}$

Our proof does not use the classical results on the eigenvalues problem for the prolate spheroidal equations as the convergent power series expansions in $\tau$ of the eigenvalues obtained by continued fractions methods in $[2]^{5}$ (cf. [2] 3.24 (10), page 240, [3], 3, page 16).

\footnotetext{
${ }^{2}$ In the line of a question asked in the conclusion of [1].

${ }^{3}$ The reader can easily check that if $\tau:=0$, then the eigenvalue $2 j(2 j+1)$ is a zero of all the polynomials $P_{2 p}$ for $p \geq j$.

${ }^{4}$ Most of our methods are perturbative, therefore we have complete results only for "small values" of the parameter $\tau$.

${ }^{5}$ Therefore, as a byproduct, we get new proofs of such results.
} 
The starting point of our proof was to try to give a rigourous meaning to a method proposed in [4], that is the "computation of the zeroes" of an infinite dimensional determinant, a Hill determinant, associated to the expansion of prolate spheroidal functions in series of Legendre polynomials, as limits of zeroes of finite dimensional determinants obtained by truncation. Our work was nearly finished when we noticed [5]. This paper contains a proof of this result for an arbitrary value of $\tau$. Our proof only works for "small values" of $\tau$, however it is necessary for the proofs of our main results.

\section{Solutions in the complex plane}

In this section, we precise the formal solutions which build basis of solutions in the neighborhood of the points $0,1,-1$ and $\infty$. Indeed, a complete description of these formal solutions can been obtained using the MAPLE package DEsiR $[6,7,8]$.

In the neighborhood of the origin, we find a basis of power series, whose first terms are the following:

$$
\begin{gathered}
y_{1}(x)=1-\frac{\mu}{2} x^{2}+\left(\frac{1}{24} \mu^{2}+\frac{1}{12} \tau^{2}-\frac{1}{4} \mu\right) x^{4}+O\left(x^{6}\right), \\
y_{2}(x)=x\left(2+\frac{-\mu+2}{3} x^{2}+\left(\frac{1}{60} \mu^{2}+\frac{1}{10} \tau^{2}-\frac{7}{30} \mu+\frac{2}{5}\right) x^{4}+O\left(x^{6}\right)\right) .
\end{gathered}
$$

In the neighborhood of each of the points \pm 1 , we have a basis of solutions constituted of a regular (holomorphic at the singularity) function $f$ and a solution of the form $f(x) \log (x \pm$ 1) $+g(x)$, where $g$ is also regular.

For example, in the neighborhood of the point 1, we obtain:

$$
\begin{gathered}
f_{1}(x)=1+\frac{-\tau^{2}+\mu}{2}(x-1)+\left(\frac{1}{16} \tau^{4}-\frac{1}{8} \mu \tau^{2}+\frac{1}{16} \mu^{2}-\frac{1}{8} \tau^{2}-\frac{1}{8} \mu\right)(x-1)^{2}+O\left((x-1)^{3}\right) \\
f_{2}(x)=\ln (x-1) f_{1}(x)+\left(-\frac{1}{2}+\tau^{2}-\mu\right)(x-1) \\
+\left(\frac{1}{8} \tau^{2}+\frac{1}{8} \mu+\frac{1}{8}-\frac{3}{16} \tau^{4}+\frac{3}{8} \mu \tau^{2}-\frac{3}{16} \mu^{2}\right)(x-1)^{2}+O\left((x-1)^{3}\right) .
\end{gathered}
$$

In general, in the neighborhood of a point $x_{0}$, the solutions are computed using the rational Newton algorithm [9], this means that we obtain "generalized formal solutions": they are parametrized by a new variable $t$ and have the form $\left(x(t)-x_{0}=\Lambda t^{r}, y(t)=\right.$ $\left.\exp (Q(1 / t)) t^{\lambda} \Phi(t)\right)$. In this expression, $\Lambda$ is a complex number (usefull in order to reduce the algebraic extension needed), $r$ is a positive integer, called the ramification, $Q$ is a polynomial without constant term, $\lambda$ is a complex number, called the exponent, and $t^{\lambda} \Phi(t)$ is the regular 
part of the formal solution. $\Phi(t)$ is a polynomial in $\log (t)$ with power series coefficients. In our example, as 1 is a regular singularity, the parametrization is only a translation $x(t)-1=$ $t$, and $Q=0$.

In the neighborhood of $\infty$, we obtain a basis of formal solutions constituted of:

$$
\left[\left[x(u)=\frac{1}{u}, y(u)=e^{-\frac{\operatorname{Rootof}\left(\tau^{2}+Z^{2}\right)}{u}} u\left(1-\frac{\operatorname{RootOf}\left(\tau^{2}+{ }_{-} Z^{2}\right)\left(\mu-\tau^{2}\right) u}{2 \tau^{2}}+\mathrm{O}\left(u^{2}\right)\right)\right]\right]
$$

Remark that we have above a condensed shape representing two solutions, corresponding to the two possible values of the Root0f: $\pm i \tau$. This gives raise to a basis of formal solutions constituted of:

$$
\begin{aligned}
& \hat{y}_{1}(x)=\frac{e^{-i \tau x}}{x}\left(1-\frac{i\left(\mu-\tau^{2}\right)}{2 \tau} \frac{1}{x}+\frac{-\mu^{2}+2 \mu+2 \tau^{2} \mu+2 \tau^{2}-\tau^{4}}{8 \tau^{2}} \frac{1}{x^{2}}+\mathrm{O}\left(\frac{1}{x^{4}}\right)\right) \\
& \hat{y}_{2}(x)=\frac{e^{i \tau x}}{x}\left(1+\frac{i\left(\mu-\tau^{2}\right)}{2 \tau} \frac{1}{x}+\frac{-\mu^{2}+2 \mu+2 \tau^{2} \mu+2 \tau^{2}-\tau^{4}}{8 \tau^{2}} \frac{1}{x^{2}}+\mathrm{O}\left(\frac{1}{x^{4}}\right)\right)
\end{aligned}
$$

The parametrization is now given by the change of variable $x=1 / u$, the ramification is trivial, and the two series which appear in these solutions are a priori divergent, but 1-summable in each direction but $\pm i \tau \mathbb{R}^{+}$.

Of course, we give here only the first terms of the series, but the following ones can be generated using a recurrence formula, so (theoretically) we can obtain as much terms as wanted in all the series appearing in the solutions.

\section{The eigenvalues as roots of an infinite determinant}

Following [[4], chapter 7, paragraph 7.5] (cf. also [5]), we try to expand a solution of the equation

$$
\mathcal{D}_{\tau}(y)=\mu y
$$

as an infinite linear combination of eigenfunctions of the unperturbated problem, i.e. the Legendre polynomials,

$$
y(x)=\sum_{n=0}^{\infty} a_{n} L_{n}(x) .
$$

We consider also $x^{2} L_{n}(x)$ as expanded in terms of the Legendre polynomials:

$$
x^{2} L_{n}(x)=\sum_{m=0}^{\infty} A_{m}^{n} L_{m}(x) .
$$


Applying the differential equation (1) to $y(x)$ and equating coefficients of $L_{n}$ for each $n$ gives an infinite matrix equation satisfied by the coefficients $a_{n}$ :

$$
M \mathbf{a}=\left(\begin{array}{ccccc}
-\mu+\tau^{2} A_{0}^{0} & \tau^{2} A_{0}^{1} & \tau^{2} A_{0}^{2} & \tau^{2} A_{0}^{3} & \ldots \\
\tau^{2} A_{1}^{0} & 2-\mu+\tau^{2} A_{1}^{1} & \tau^{2} A_{1}^{2} & \tau^{2} A_{1}^{3} & \ldots \\
\tau^{2} A_{2}^{0} & \tau^{2} A_{2}^{1} & 6-\mu-\tau^{2} A_{2}^{2} & \tau^{2} A_{2}^{3} & \ldots \\
\vdots & \vdots & \vdots & \vdots & \ddots
\end{array}\right)\left(\begin{array}{c}
a_{0} \\
a_{1} \\
a_{2} \\
\vdots
\end{array}\right)=0
$$

In our case, it is possible to obtain explicit formulas for the coefficients $A_{m}^{n}$. Indeed, $A_{m}^{n}=\frac{1}{m+1 / 2} \int_{-1}^{1} x^{2} L_{n}(x) L_{m}(x) d x$. Using the recurrence relation

$$
(2 n+1) x L_{n}=(n+1) L_{n+1}+n L_{n-1}
$$

and the fact that $\int_{-1}^{1} L_{n}(x) L_{m}(x) d x=\frac{2 \delta_{m}^{n}}{2 n+1}$, we obtain:

$$
A_{n}^{n}=\frac{2 n^{2}+2 n-1}{(2 n+3)(2 n-1)}, A_{n}^{n+2}=\frac{(n+1)(n+2)}{(2 n+1)(2 n+3)}, A_{n+2}^{n}=\frac{(n+1)(n+2)}{(2 n+3)(2 n+5)} .
$$

Then the matrix $M$ is tridiagonal:

$$
M=\left(\begin{array}{cccccc}
-\mu+\tau^{2} / 3 & 0 & 2 \tau^{2} / 3 & 0 & 0 & \ldots \\
0 & 2-\mu+3 \tau^{2} / 5 & 0 & 2 \tau^{2} / 5 & 0 & \ldots \\
2 \tau^{2} / 15 & 0 & 6-\mu+11 \tau^{2} / 21 & 0 & 12 \tau^{2} / 35 & \ldots \\
0 & 6 \tau^{2} / 35 & 0 & 12-\mu+23 \tau^{2} / 45 & 0 & \ldots \\
0 & 0 & 4 \tau^{2} / 21 & 0 & 20-\mu+39 \tau^{2} / 77 & \ldots \\
\vdots & \vdots & \vdots & \vdots & \vdots & \ddots
\end{array}\right) .
$$

Moreover, we can split the odd and even coefficients $a_{n}$, by extracting from the matrix $M$ the corresponding columns and rows.

For example, the even coefficients satisfy the following system:

$$
\left(\begin{array}{cccc}
-\mu+\tau^{2} / 3 & 2 \tau^{2} / 3 & 0 & \ldots \\
2 \tau^{2} / 15 & 6-\mu+11 \tau^{2} / 21 & 12 \tau^{2} / 35 & \ldots \\
0 & 4 \tau^{2} / 21 & 20-\mu+39 \tau^{2} / 77 & \ldots \\
\vdots & \vdots & \vdots & \ddots
\end{array}\right)\left(\begin{array}{c}
a_{0} \\
a_{2} \\
a_{4} \\
\vdots
\end{array}\right)=0 .
$$

Next we truncate the previous infinite matrix, and define $M^{(n)}$ the $n \times n$ matrix whose entries are the same as the first $n$ rows and columns of the infinite matrix. 
We define $D_{n}$ as the determinant of $M^{(n)} . D_{n}$ is a polynomial in the variables $\mu$ and $\tau^{2}$, of $n$ th-order in the variable $\mu$. Considering the special shape of the matrix $M^{(n)}$, it is easy to derive the recurrence formula:

$$
D_{n+1}=\left(2 n(2 n+1)-\mu+A_{2 n}^{2 n} \tau^{2}\right) D_{n}-A_{2 n}^{2 n-2} A_{2 n-2}^{2 n} \tau^{4} D_{n-1} .
$$

So we obtain a family of polynomials, defined by the initial values

$$
D_{0}=1, D_{1}=-\mu+\frac{1}{3} \tau^{2},
$$

and a recurrence equation, whose roots are good candidates to approach the exact eigenvalues of even index of the differential equation (1).

Proposition 1. The polynomial $D_{n}$ is a polynomial of degree $n$ in the variable $\mu$. For all $n \geq 1$,

$$
D_{n} \quad \bmod \tau^{2}=(-1)^{n} \prod_{j=0}^{n-1}(\mu-2 j(2 j+1))
$$

Then $D_{n} \bmod \tau^{2}$ admits $n$ (integer) roots: $0,6, \ldots, 2 j(2 j+1), \ldots,(2 n-2)(2 n-1)$, all are simple.

The polynomial $D_{n}$ admits $n$ Puiseux series solutions, we note $\mu_{2 j}^{(n)}$ the series, which is equal to $2 j(2 j+1) \bmod \tau^{2}$.

Proof: Perform the reduction of $D_{n}$ modulo $\tau^{2}$ :

$$
\left(D_{j+1} \bmod \tau^{2}\right)=(2 j(2 j+1)-\mu)\left(D_{j} \bmod \tau^{2}\right) .
$$

Proposition 2. Convergence radii of the series $\mu_{2 j}^{(n)}$.

The series $\mu_{2 j}^{(n)}, j \geq 0$ converge for $|\tau|<1$.

Proof: The following result can be found in [[10], p. 95]:

Let $X$ be a unitary space, let $T(x)=T+x T^{(1)}$ a linear operator on $X$ and let $T$ be normal. Then the power series for the eigenvalues $\lambda(x)$ are convergent if "the magnitude of the perturbation" $\left\|x T^{(1)}\right\|$ is smaller than half the isolation distance of the eigenvalue $\lambda$ of $T$.

More precisely, if $\lambda(x)=\sum_{p \geq 0} \lambda_{p} x^{p}$, the coefficients $\lambda_{p}$ satisfy the majorations:

$$
\left|\lambda_{1}\right| \leq a, \quad\left|\lambda_{p}\right| \leq a^{p}\left(\frac{2}{d}\right)^{p-1}, p \geq 2,
$$

where $a=\left\|T^{(1)}\right\|$ and $d$ is the isolation distance of the eigenvalues of $T$.

We apply this result here by searching the eigenvalues of:

$$
F^{(n)}+\tau^{2} G^{(n)}
$$


where

$$
F^{(n)}=\left(\begin{array}{lllll}
0 & & & & \\
& 6 & & & \\
& & & & \\
& 20 & & \\
& & \ddots & \\
& & & & 2(n-1)(2 n-1)
\end{array}\right),
$$

and

$$
G^{(n)}=\left(\begin{array}{ccccc}
1 / 3 & 2 / 3 & & & \\
2 / 15 & 11 / 21 & 12 / 35 & & \\
& 4 / 21 & 39 / 77 & 10 / 33 & \\
& & \ddots & \ddots & \ddots \\
& & & A_{2 n-2}^{2 n-4} & A_{2 n-2}^{2 n-2}
\end{array}\right)
$$

The matrices $F^{(n)}$ and $G^{(n)}$ are $n \times n$ square matrices, $G^{(n)}$ is a tridiagonal matrix, whose elements are explicitely known.

It is easy to majorate the 2-norm of $G^{(n)}$, using the inequality $\left\|G^{(n)}\right\|_{2} \leq$ $\sqrt{\left\|G^{(n)}\right\|_{1}\left\|G^{(n)}\right\|_{\infty}} \leq 3$.

We obtain the announced result by noting that $d=6$ (for all $n$ ).

In the following paragraph, we will study the link between the series $\mu_{2 j}^{(n)}$, when $j$ is fixed and $n$ is growing.

\section{The eigenvalues as sums of a series}

First we compute the beginning of the Puiseux series of the polynomials $D_{n}$, for small $n$.

$$
\begin{aligned}
& >\quad \text { algcurves [puiseux] (D (1) , tau }=0, \mathrm{mu}, 9) ; \\
& \qquad\left\{\frac{1}{3} \tau^{2}\right\} \\
& >\quad \text { algcurves [puiseux] (D (2), tau }=0, \mathrm{mu}, 9) ; \\
& \quad\left\{\frac{1}{3} \tau^{2}-\frac{2}{135} \tau^{4}+\frac{4}{8505} \tau^{6}+\frac{58}{2679075} \tau^{8}, 6+\frac{11}{21} \tau^{2}+\frac{2}{135} \tau^{4}-\frac{4}{8505} \tau^{6}-\frac{58}{2679075} \tau^{8}\right\} \\
& >\quad \text { algcurves }[\text { puiseux] (D (3), tau }=0, \mathrm{mu}, 9) ;
\end{aligned}
$$




$$
\begin{aligned}
& \left\{\frac{1}{3} \tau^{2}-\frac{2}{135} \tau^{4}+\frac{4}{8505} \tau^{6}+\frac{26}{1913625} \tau^{8}, 6+\frac{11}{21} \tau^{2}+\frac{94}{9261} \tau^{4}-\frac{21388}{44925111} \tau^{6}-\frac{2946550}{217931713461} \tau^{8},\right. \\
& \left.20+\frac{39}{77} \tau^{2}+\frac{8}{1715} \tau^{4}+\frac{16}{2773155} \tau^{6}-\frac{2476}{37368263625} \tau^{8}\right\} \\
& >\quad \text { algcurves [puiseux] (D (4), tau=0,mu, 9); } \\
& \left\{\frac{1}{3} \tau^{2}-\frac{2}{135} \tau^{4}+\frac{4}{8505} \tau^{6}+\frac{26}{1913625} \tau^{8}, 6+\frac{11}{21} \tau^{2}+\frac{94}{9261} \tau^{4}-\frac{21388}{44925111} \tau^{6}-\frac{3633830}{2575556661363} \tau^{8},\right. \\
& 20+\frac{39}{77} \tau^{2}+\frac{52674}{29674645} \tau^{4}+\frac{935076}{175940970205} \tau^{6}+\frac{37764832611642}{74924536936711587125} \tau^{8}, \\
& \left.42+\frac{83}{165} \tau^{2}+\frac{60134}{76366125} \tau^{4}+\frac{101060636}{276516011165625} \tau^{6}+\frac{504082644490258}{6322139029665881296875} \tau^{8}\right\}
\end{aligned}
$$

We notice that the first terms of the series stabilize little by little.

More precisely, consider the "first" series solution of all polynomials, the series whose constant term is null. The term of degree 2 is the same for all poynomials, the terms of degree 4 and 6 are the same for all polynomials of index greater than 1, the terms of degrre 6 and 8 are the same for all polynomial of index greater than 2 .

This phenomenon is repeated for the "second" series solution, the series whose constant term is 6 . The term of degree 2 is the same for all polynomials of index greater than 1 , the terms of degree 4 and 6 are the same for all polynomials of index greater than 2 , etc...

We can express that in the following way:

if we put $\mu_{0}=0, D_{1}\left(\mu_{0}\right)=0 \bmod \tau^{2}, \mu_{1}=\frac{\tau^{2}}{3}, D_{1}\left(\mu_{1}\right)=0 \bmod \tau^{4} ; \mu_{2}=\mu_{1}-\frac{2}{135} \tau^{4}+$ $\frac{4}{8505} \tau^{6}, D_{2}\left(\mu_{2}\right)=0 \bmod \tau^{8}$.

Also, if $\mu_{0}=6, D_{2}\left(\mu_{0}\right)=0 \bmod \tau^{2}, \mu_{1}=6+\frac{11}{21} \tau^{2}, D_{2}\left(\mu_{1}\right)=0 \bmod \tau^{4} ; \mu_{2}=\mu_{1}+\frac{94}{9261} \tau^{4}-$ $\frac{21388}{44925111} \tau^{6}, D_{3}\left(\mu_{2}\right)=0 \bmod \tau^{8}$.

And also, if $\mu_{0}=20, D_{3}\left(\mu_{0}\right)=0 \bmod \tau^{2}, \mu_{1}=20+\frac{39}{77} \tau^{2}, D_{3}\left(\mu_{1}\right)=0 \bmod \tau^{4}$.

So we can prove the

Proposition 3. Let $j \geq 0$. We put $\mu_{0}=2 j(2 j+1)$. Then $D_{j+1}\left(\mu_{0}\right)=0 \bmod \tau^{2}$. We build $\mu_{1}=\mu_{0}-\frac{D_{j+1}\left(\mu_{0}\right)}{D_{j+1}^{\prime}\left(\mu_{0}\right)} \bmod \tau^{4}$ and we prove that $D_{j+1}\left(\mu_{1}\right)=0 \bmod \tau^{4}$.

More generally, for all $i \geq 1$, if $\mu_{i}=\mu_{i-1}-\frac{D_{j+i}\left(\mu_{i-1}\right)}{D_{j+i}^{\prime}\left(\mu_{i-1}\right)} \bmod \tau^{4 i}$, then

$$
D_{j+i}\left(\mu_{i}\right)=0 \quad \bmod \tau^{4 i}
$$

Proof: From above, it is clear that $D_{j+1}\left(\mu_{0}\right)=0 \bmod \tau^{2}$ and that $\left(D_{j+1} \bmod \tau\right)^{\prime}\left(\mu_{0}\right) \neq$ 0 . So $D_{j+1}^{\prime}\left(\mu_{0}\right)$ is a polynomial in $\tau$ with a non nul constant term, which means that it is invertible in the ring of formal series, then $\mu_{1}$ is well defined. Moreover $\mu_{1}=\mu_{0} \bmod \tau^{2}$. We recall the following Taylor formula [[11], p. 51]: let $a$ be a univariate polynomial over an arbitrary integral domain $\mathcal{A}$. In the polynomial domain $\mathcal{A}[x, y], a(x+y)=a(x)+a^{\prime}(x) y+$ 
$b(x, y) y^{2}$ for some polynomial $b \in \mathcal{A}[x, y]$. We apply this result with $a=D_{j+1}$ and $\mathcal{A}=\mathbb{Q}[\tau]:$

$$
\begin{aligned}
D_{j+1}\left(\mu_{1}\right) & =D_{j+1}\left(\mu_{0}\right)+\left(\mu_{1}-\mu_{0}\right) D_{j+1}^{\prime}\left(\mu_{0}\right)+\left(\mu_{1}-\mu_{0}\right)^{2} b\left(\mu_{0}, \mu_{1}\right) \\
& =D_{j+1}\left(\mu_{0}\right)+\left(\mu_{1}-\mu_{0}\right) D_{j+1}^{\prime}\left(\mu_{0}\right) \bmod \tau^{4}=0 \bmod \tau^{4} .
\end{aligned}
$$

We assume now that we have built the first terms until the index $i-1$ (for some $i \geq 2$ ). By construction: $\mu_{i-1}=\mu_{0} \bmod \tau^{2}$, then $D_{j+i}^{\prime}\left(\mu_{i-1}\right) \bmod \tau=\left(D_{j+i} \bmod \tau\right)^{\prime}\left(\mu_{i-1}\right.$ $\bmod \tau)=\left(D_{j+i} \bmod \tau\right)^{\prime}\left(\mu_{0}\right)$ is not null. Then $\mu_{i}$ is well defined. Moreover, by using the recurrence equation and the fact that $D_{j+i-1}\left(\mu_{i-1}\right)=0 \bmod \tau^{4(i-1)}$ and that $D_{j+i-2}\left(\mu_{i-1}\right)$ $\bmod \tau^{4(i-2)}=D_{j+i-2}\left(\mu_{i-2}\right) \bmod \tau^{4(i-2)}=0$, we obtain that $D_{j+i}\left(\mu_{i-1}\right)=0 \bmod \tau^{4(i-1)}$, thus $\mu_{i}=\mu_{i-1} \bmod \tau^{4(i-1)}$.

The same Taylor formula enables us to prove that $D_{j+i}\left(\mu_{i}\right)=0 \bmod \tau^{4 i}$.

In fact, $\mu_{i}$ is built by adding to $\mu_{i-1}$ two monomials, of degree $4 i-4$ and $4 i-2$, and from a computing point of view $\mu_{i}=\mu_{i-1}-\frac{D_{j+i}\left(\mu_{i-1}\right)}{D_{j+i}^{\prime}\left(\mu_{1}\right)} \bmod \tau^{4 i}$.

Proposition 4. For all $j \geq 0$, we have built a formal series in the variable $\tau, \hat{\mu}_{2 j}$, such that

$$
\hat{\mu}_{2 j}=2 j(2 j+1) \bmod \tau^{2} \text { et } \forall n>j, D_{n}\left(\hat{\mu}_{2 j}\right)=0 \bmod \tau^{4(n-j)} .
$$

To fix the ideas, we give the first terms of the series $\hat{\mu}_{0}, \hat{\mu}_{2}, \hat{\mu}_{4}$.

$$
\begin{aligned}
& \hat{\mu}_{0}=\frac{1}{3} \tau^{2}-\frac{2}{135} \tau^{4}+\frac{4}{8505} \tau^{6}+\frac{26}{1913625} \tau^{8}-\frac{92}{37889775} \tau^{10}+\frac{513988}{9050920003125} \tau^{12} \\
& \hat{\mu}_{2}=6+\frac{11}{21} \tau^{2}+\frac{94}{9261} \tau^{4}-\frac{21388}{44925111} \tau^{6}-\frac{3633830}{257555661363} \tau^{8}+\frac{39611204}{16226006665869} \tau^{10} \\
& -\frac{277773545116}{4906403669618802351} \tau^{12} \\
& \hat{\mu}_{4}=20+\frac{39}{77} \tau^{2}+\frac{52674}{29674645} \tau^{4}+\frac{935076}{175940970205} \tau^{6}+\frac{37764832611642}{74924536936711587125} \tau^{8} \\
& -\frac{3187867616210148}{241152114584499914320525} \tau^{10}-\frac{16139900980217820949844}{90612997487168811993405138946875} \tau^{12}
\end{aligned}
$$

In the following paragraphs, we will apply the following lemma:

Lemma 1. Let $P$ be a polynomial, $P \in \mathbb{C}[\tau][y], \mu^{*}$ a series $(\in \mathbb{C}[[\tau]])$ satisfying $P\left(\mu^{*}\right)=0$, $P^{\prime}\left(\mu^{*}\right) \neq 0$ and $\hat{\mu}$ an other series such that $P(\hat{\mu})=0 \bmod \tau^{k}$ and $\hat{\mu}=\mu^{*} \bmod \tau$. Then $\hat{\mu}=\mu^{*} \bmod \tau^{k}$.

Proof: We use the above Taylor formula [[11], p. 51].

Proposition 5. Link between the series $\mu_{2 j}^{(n)}$ and the series $\hat{\mu}_{2 j}$

$$
\forall j \geq 0, \forall n>j, \quad \mu_{2 j}^{(n)}=\hat{\mu}_{2 j} \quad \bmod \tau^{4(n-j)} .
$$


Proof: By construction of the series $\hat{\mu}_{2 j}$ or by applying the lemma.

TheOREM 1. For all $j \geq 0$, the series $\hat{\mu}_{2 j}$ is convergent in the open unit disk, and for all $|\tau|<1$, the sequence $\mu_{2 j}^{(n)}(\tau)$ converges, when $n$ tends to infinity, to $\hat{\mu}_{2 j}(\tau)$, which is an eigenvalue $\mathcal{D}_{\tau}$.

Proof: Let $\alpha_{p}^{(n)}$ be the coefficients of the series $\mu_{2 j}^{(n)}$ and $\alpha_{p}$ those of $\hat{\mu}_{2 j}$. The majoration given by Kato is independent of $n$, because $d$ is independent of $n$ and $\left\|G^{(n)}\right\|_{2}$ is bounded independently of $n:\left|\alpha_{p}^{(n)}\right| \leq 3$ (for all $n$ and for all $p$ ). So we have also $\left|\alpha_{p}\right| \leq 3$ (for all $p$ ). Let $|\tau|<1$.

$$
\mu_{0}^{(n)}(\tau)-\hat{\mu}_{0}(\tau)=\sum_{p>4 n}\left(\alpha_{p}^{(n)}-\alpha_{p}\right) \tau^{2 p}
$$

and

$$
\left|\mu_{0}^{(n)}(\tau)-\hat{\mu}_{0}(\tau)\right| \leq 6 \sum_{p>4 n}\left|\tau^{2 p}\right|,
$$

what we can make arbitrarily small when $n$ tends to infinity.

The last part of the theorem, the fact that $\hat{\mu}_{2 j}(\tau)$ is an eigenvalue of $\mathcal{D}_{\tau}$, will be proved in the paragraph 9. It is also possible to prove this result using [5] but we propose a self-contained proof for all our results.

\section{The eigenvalues and the coordinates of the eigenfunctions in Legendre basis}

Consider an eigenfunction of even index of the equation $\mathcal{D}_{\tau}(y)=\mu y$ as an infinite linear combination of the Legendre polynomials,

$$
y(x)=\sum_{n=0}^{\infty} a_{2 n} L_{2 n}(x), \quad a_{0}=1 .
$$

We will now see that there exists a simple relation between $a_{2 n}$ and $D_{n}$.

Proposition 6.

$$
\forall n \geq 1, \quad D_{n}=(-1)^{n}\left(\prod_{i=1}^{n} A_{2 i-2}^{2 i}\right) \tau^{2 n} a_{2 n} .
$$

Proof: By recurrence.

For $n=1: a_{2}$ is defined by the equation $D_{1} a_{0}+A_{0}^{2} \tau^{2} a_{2}=0$.

Suppose that the relation is satisfied for a fixed integer $n \geq 1$. We perform the Gaussian elimination algorithm, in order to put the matrix $M^{(n+2)}$ in an echelon form. 
We assume that the first steps give the intermediate result:

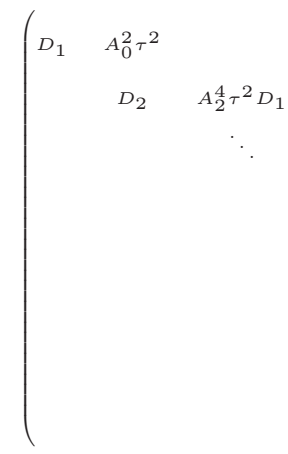

$$
\begin{aligned}
& D_{j} \quad A_{2 j-2}^{2 j} \tau^{2} D_{j-1} \\
& A_{2 j}^{2 j-2} \tau^{2} \quad 2 j(2 j+1)-\mu+A_{2 j}^{2 j} \tau^{2} \quad A_{2 j}^{2 j+2} \tau^{2} \\
& A_{2 n}^{2 n-2} \tau^{2} \quad 2 n(2 n+1)-\mu+A_{2 n}^{2 n} \tau^{2} \\
& A_{2 n+2}^{2 n} \tau^{2}
\end{aligned}
$$$$
A_{2 n}^{2 n+2} \tau^{2}
$$$$
\left.(2 n+2)(2 n+3)-\mu+A_{2 n+2}^{2 n+2} \tau^{2}\right)
$$

The next step is: replace $\operatorname{row}_{j+1}$ by $D_{j}$ row $_{j+1}-A_{2 j}^{2 j-2} \tau^{2}$ row $_{j}$; using the recurrence relation giving $D_{j+1}$ in terms of $D_{j}$ and $D_{j-1}$, we obtain:

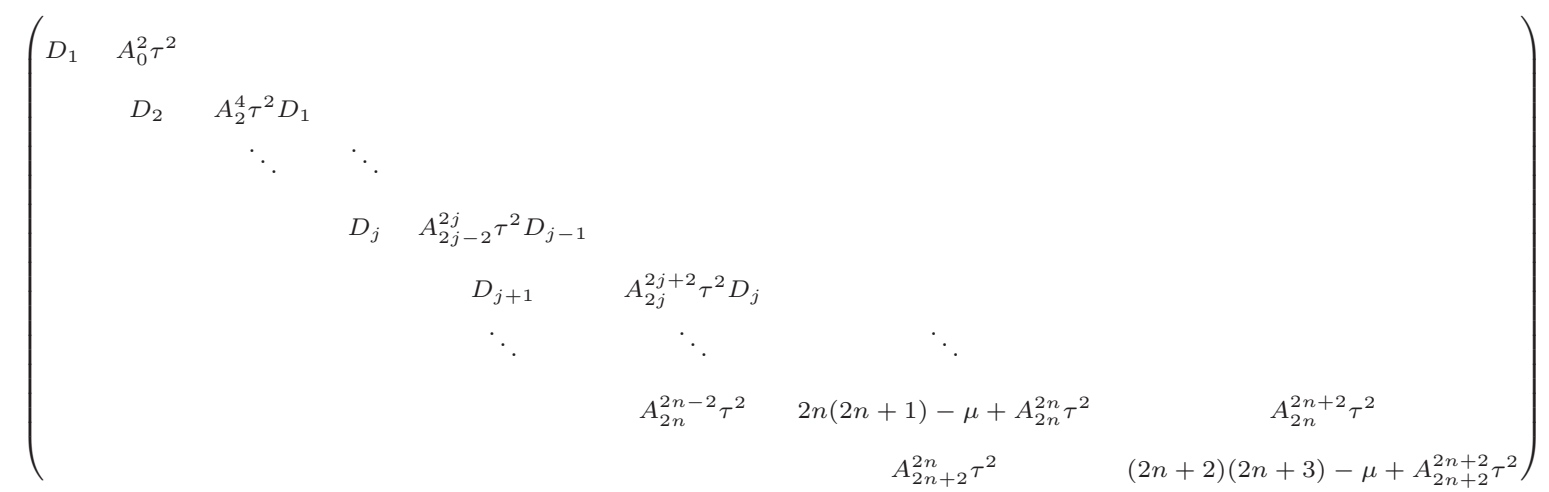

Then, at the final step, we will have:

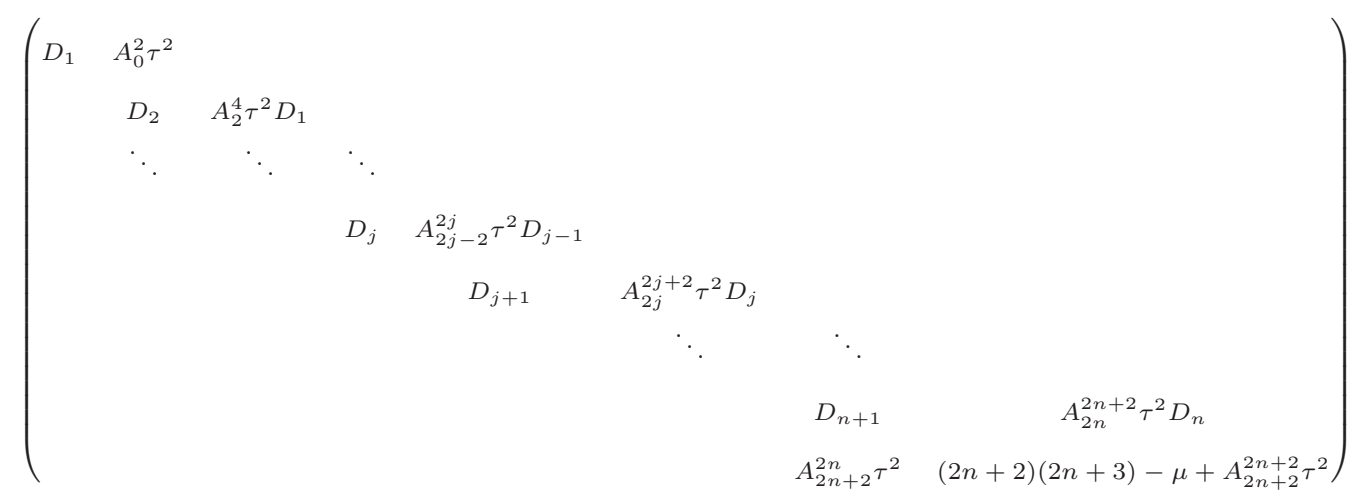

The penultimate row gives:

$$
D_{n+1} a_{2 n}+A_{2 n}^{2 n+2} \tau^{2} D_{n} a_{2 n+2}=0,
$$

which is the expected relation for $n+1$. 
Corollary 1: for all $n \geq 1$, the coefficient $a_{2 n}$ has the same zeros as the polynomial $D_{n}$. Thus, for all $|\tau|<1$, the zero of index $j$ of the coefficient $a_{2 n}$ converges to $\hat{\mu}_{2 j}(\tau)$, when $n$ tends to infinity.

Proposition 7. For all $j \geq 0$, the series $\hat{\mu}_{2 j}$ satisfies :

$$
\forall n>2 j, \quad a_{2 n}\left(\hat{\mu}_{2 j}\right)=0 \quad \bmod \tau^{2(n-2 j)} .
$$

\section{The eigenvalues and the formal solutions at the origin}

We recall that, in the neighborhood of the origin, we know a basis of convergent series solutions, $y_{1}(x)$ et $y_{2}(x)$.

In particular:

$$
y_{1}(x)=1-\frac{\mu}{2} x^{2}+\left(\frac{1}{24} \mu^{2}+\frac{1}{12} \tau^{2}-\frac{1}{4} \mu\right) x^{4}+O\left(x^{6}\right) .
$$

Let $P_{n}$ be the coordinates of this function in the monomial basis:

$$
P_{0}=1, P_{1}=0, P_{2}=-\frac{\mu}{2}, P_{3}=0, P_{4}=\frac{1}{24} \mu^{2}+\frac{1}{12} \tau^{2}-\frac{1}{4} \mu,
$$

the following polynomials satisfying a three terms recurrence:

$$
-n(n-1) P_{n}+\left(n^{2}-\mu-3 n+2\right) P_{n-2}+\tau^{2} P_{n-4}=0 .
$$

Proposition 8. The odd coordinates are null. The even coordinates, $P_{2 n}$ are polynomials of degree $n$ in the variable $\mu$ and for all $n \geq 1, P_{2 n} \bmod \tau^{2}=\frac{(-1)^{n}}{(2 n) !} \prod_{j=0}^{n-1} \mu-2 j(2 j+1)$. The polynomial $P_{2 n}$ admits $n$ Puiseux series solutions, we will denote $\delta_{j}^{(n)}, j=0 \ldots n-1$, the series which has $2 j(2 j+1)$ as constant term.

Proof: Reduce the recurrence equation modulo $\tau^{2}$ :

$$
2 n(2 n-1)\left(P_{2 n} \quad \bmod \tau^{2}\right)=((2 n-1)(2 n-2)-\mu)\left(P_{2 n-2} \quad \bmod \tau^{2}\right) .
$$

Proposition 9. For all $j \geq 0$, the series $\hat{\mu}_{2 j}$ satisfies:

$$
\forall n>j, \quad P_{2 n}\left(\hat{\mu}_{2 j}\right)=0 \quad \bmod \tau^{2(n-j)} .
$$

Proof: Taking into account the parity of the functions, the eigenfunction of even index that we consider:

$$
y(x)=\sum_{n=0}^{\infty} a_{2 n} L_{2 n}(x), \quad a_{0}=1
$$


is proportional to $y_{1}$, that is $y(x)=c y_{1}(x)$.

We obtain by change of basis:

$$
\left(\begin{array}{c}
c \\
c P_{2} \\
c P_{4} \\
\vdots
\end{array}\right)=A\left(\begin{array}{c}
1 \\
a_{2} \\
a_{4} \\
\vdots
\end{array}\right)
$$

where we only need to note that $A$ is an infinite upper triangular matrix, whose elements will be noted $\alpha_{i j}$.

$$
A=\left(\begin{array}{cccc}
1 & -1 / 2 & 3 / 8 & \ldots \\
& 3 / 2 & -15 / 4 & \ldots \\
& & 35 / 8 & \ldots \\
& & & \ddots
\end{array}\right) .
$$

Then: $c=\sum_{j=0}^{\infty} \alpha_{1 j} a_{2 j}$ and, using the proposition $7, c\left(\hat{\mu}_{0}\right)=1 \bmod \tau^{2}$. We deduce:

$$
c\left(\hat{\mu}_{0}\right) P_{2 n}\left(\hat{\mu}_{0}\right)=\alpha_{n n} a_{2 n}\left(\hat{\mu}_{0}\right) \bmod \tau^{2 n+2}=0 \bmod \tau^{2 n}, \forall n \geq 1 .
$$

Also $\tau^{2} c\left(\hat{\mu}_{2}\right)=\tau^{2}-\frac{1}{2} \tau^{2} a_{2}\left(\hat{\mu}_{2}\right)+\frac{3}{8} \tau^{2} a_{4}\left(\hat{\mu}_{2}\right) \bmod \tau^{4}=\frac{1}{2 A_{0}^{2}} D_{1}\left(\hat{\mu}_{2}\right) \bmod \tau^{2} \neq 0 \bmod \tau$.

We deduce: $\tau^{2} c\left(\hat{\mu}_{2}\right) P_{4}\left(\hat{\mu}_{2}\right)=-\frac{35}{8} \tau^{2} a_{4}\left(\hat{\mu}_{2}\right) \bmod \tau^{4}=-\frac{35}{8 A_{0}^{2} A_{2}^{4} \tau^{2}} D_{2}\left(\hat{\mu}_{2}\right) \bmod \tau^{4}=0$ $\bmod \tau^{2}$, hence $P_{4}\left(\hat{\mu}_{2}\right)=0 \bmod \tau^{2}$.

And $\forall n \geq 2$,

$$
\tau^{2} c\left(\hat{\mu}_{2}\right) P_{2 n}\left(\hat{\mu}_{2}\right)=\alpha_{n n} \tau^{2} a_{2 n}\left(\hat{\mu}_{2}\right) \quad \bmod \tau^{2 n}=\frac{c s t e}{\tau^{2(n-1)}} D_{n}\left(\hat{\mu}_{2}\right) \quad \bmod \tau^{2 n}=0 \quad \bmod \tau^{2(n-1)},
$$

then $P_{2 n}\left(\hat{\mu}_{2}\right)=0 \bmod \tau^{2(n-1)}, \forall n \geq 2$.

In a general way:

$$
\begin{aligned}
\tau^{2 j} c\left(\hat{\mu}_{2 j}\right) & =\sum_{i=0} \alpha_{1 i} \tau^{2 j} a_{2 i}\left(\hat{\mu}_{2 j}\right)=\sum_{i=0}^{2 j} \alpha_{1 i} \tau^{2 j} a_{2 i}\left(\hat{\mu}_{2 j}\right) \quad \bmod \tau^{2 j+2} \\
& =\alpha_{1 j} \frac{(-1)^{j}}{\prod_{k=1}^{j} A_{2 k-2}^{2 k}} D_{j}\left(\hat{\mu}_{2 j}\right) \quad \bmod \tau^{2} \neq 0 \quad \bmod \tau .
\end{aligned}
$$


We deduce:

$$
\begin{aligned}
\forall n>j, \tau^{2 j} c\left(\hat{\mu}_{2 j}\right) P_{2 n}\left(\hat{\mu}_{2 j}\right) & =\alpha_{n n} \tau^{2 j} a_{2 n}\left(\hat{\mu}_{2 j}\right) \bmod \tau^{2(n+1-j)} \\
& =\frac{c s t e}{\tau^{2(n-j)}} D_{n}\left(\hat{\mu}_{2 j}\right) \bmod \tau^{2(n+1-j)}=0 \bmod \tau^{2(n-j)},
\end{aligned}
$$

then $P_{2 n}\left(\hat{\mu}_{2 j}\right)=0 \bmod \tau^{2(n-j)}, \forall n>j$.

Proposition 10. Link between the series $\delta_{j}^{(n)}$ and $\hat{\mu}_{2 j}$.

$$
\forall j \geq 0, \forall n>j, \quad \delta_{j}^{(n)}=\hat{\mu}_{2 j} \quad \bmod \tau^{2(n-j)} .
$$

Proof: Apply lemma 1.

TheOREM 2. For $\tau$ small enough, the sequence $\delta_{j}^{(n)}(\tau)$ converges to $\hat{\mu}_{2 j}(\tau)$, when $n$ tends to infinity.

Proof: To obtain this result, we will first prove that the radius of convergence of the series $\delta_{j}^{(n)}$ is bounded from below by a constant independent of $n$.

The recurrence equation satisfied by the polynomials $P_{n}$ can be written, noting $\mathbb{P}_{n}=P_{2 n}$ :

$$
\tau^{2} \mathbb{P}_{n-1}+(2 n(2 n+1)-\mu) \mathbb{P}_{n}-(2 n+1)(2 n+2) \mathbb{P}_{n+1}=0 .
$$

Thus $\mathbb{P}_{n+1}(\mu)=0$ if and only if

$$
\left(\begin{array}{ccccccc}
-\mu & -2 & & & & & \\
\tau^{2} & 6-\mu & -12 & & & & \\
& \ddots & \ddots & \ddots & & & \\
& & \tau^{2} & 2 k(2 k+1)-\mu & -2 k(2 k-1) & & \\
& & & \ddots & \ddots & \ddots & \\
& & & & \tau^{2} & 2(n-1)(2 n-1)-\mu & -2(n-1)(2 n-3) \\
& & & & & \tau^{2} & 2 n(2 n+1)-\mu
\end{array}\right)\left(\begin{array}{c}
\mathbb{P}_{0} \\
\mathbb{P}_{1} \\
\vdots \\
\mathbb{P}_{k} \\
\vdots \\
\mathbb{P}_{n-1} \\
\mathbb{P}_{n}
\end{array}\right)=0 .
$$

So we are again concerned with the eigenvalues of a perturbated matrix:

$$
F^{(n)}+\tau^{2} G^{(n)},
$$

where

$$
F^{(n)}=\left(\begin{array}{ccccc}
0 & -2 & & & \\
& 6 & -12 & & \\
& \ddots & \ddots & \\
& & 2(n-1)(2 n-1) & -2(n-1)(2 n-3) \\
& & & 2 n(2 n+1)
\end{array}\right), \quad G^{(n)}=\left(\begin{array}{cccc}
0 & & & \\
1 & 0 & & \\
& & \ddots & \ddots \\
& & 1 & 0
\end{array}\right) .
$$


The result used in the proof of propositon 2 can not be applied here, because the new matrix $F^{(n)}$ is not normal.

Neverthless, we can follow the developments of Kato, [[10], p. 88-90, example 3.3] to evaluate the radii of convergence of the series $\delta_{j}^{(n)}$ in the following manner: we compute the resolvant of the matrix $F^{(n)}$ and its expansion in the neighborhood of its eigenvalue $\mu=2 j(2 j+1)$ $(j \leq n)$. This expansion can be written:

$$
R^{(n)}(\zeta)=\left(F^{(n)}-\zeta I_{n}\right)^{-1}=\frac{-1}{\zeta-\mu} T^{(n)}+\sum_{k=0}^{\infty}(\zeta-\mu)^{k}\left(S^{(n)}\right)^{k+1} .
$$

Then we denote $p_{n}=\left\|G^{(n)} T^{(n)}\right\|_{1}, q_{n}=\left\|G^{(n)} S^{(n)}\right\|_{1}, s_{n}=\left\|S^{(n)}-\alpha T^{(n)}\right\|_{1}$ (for any $\alpha$ ). Using methods of majorant series, Kato proves that $\left(\left(p_{n} s_{n}\right)^{1 / 2}+q_{n}^{1 / 2}\right)^{-2}$ is a lower bound for the radius of convergence of the series $\delta_{j}^{(n)}$.

Our problem is thus to prove that $p_{n}, q_{n}$ and $s_{n}$ can be bounded from above independently of $n$.

Now the resolvant $R^{(n)}(\zeta)$ is an upper triangular matrix, whose elements can be computed explicitely; these elements don't depend from the index $n$ and we will omit the upper index $(n)$ to simplify the notations:

$$
R_{i l}(\zeta)=(-1)^{l-i+1} \frac{(2(l-1)) !}{(2(i-1)) !} \prod_{k=i-1}^{l-1} \frac{1}{\zeta-2 k(2 k+1)}
$$

This enables us to compute explicitely the matrix $T^{(n)}$ : it means computing the polar part of the rational fractions $R_{i l}$ at the pole $\mu$. This one is null if $i>j+1$ or $l \leq j$. Thus the first $j$ columns of the matrix $T^{(n)}$ are null, and we can find non null elements only on the first $j+1$ rows.

For $i \leq j+1$ and $l>j, T_{i l}=-R_{i l}(\zeta) \times(\zeta-\mu)$ evaluated in $\mu$. Therefore

$$
T_{i l}=(-1)^{l-i} \frac{(2(l-1)) !}{(2(i-1)) !} \prod_{k \geq i-1, k \neq j}^{l-1} \frac{1}{\mu-2 k(2 k+1)} .
$$

Thus $T_{i l+1}=\frac{2 l(2 l-1)}{2 l(2 l+1)-\mu} T_{i l}$. For all $j$, there exists an index $l_{0}$ from which the ratio $\frac{2 l(2 l-1)}{2 l(2 l+1)-\mu}$ is lower than 1 ; from this index, the 1-norm of the columns of $T^{(n)}$ is decreasing. Finally, considering the structure of $G^{(n)},\left(G^{(n)} T^{(n)}\right)_{i l}=T_{i-1 l}$. We conclude that there exists an upper bound for $p_{n}$, independent of $n$.

It follows from above that the coefficients $T_{i l}$ are bounded, we will denote $\|T\|^{\infty}$ a bound for $\left|T_{i l}\right|$. 
For the matrix $S$, we compute the constant term in the expansion of the rational fractions $R_{i l}$ in the neighborhood of $\mu$. The matrix $S$ has the following structure:

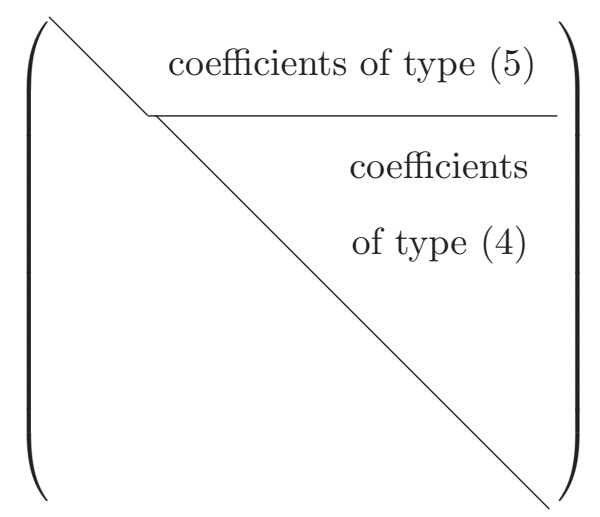

For $i>j+1, \mu$ is not a pole, thus $S_{i l}=R_{i l}(\mu)$, that means:

$$
S_{i l}=(-1)^{l-i+1} \frac{(2(l-1)) !}{(2(i-1)) !} \prod_{k=i-1}^{l-1} \frac{1}{\mu-2 k(2 k+1)}
$$

if $l \geq i$ and else 0 .

As before, $S_{i l+1}=\frac{2 l(2 l-1)}{2 l(2 l+1)-\mu} S_{i l}$ if $l \geq i, S_{l+1 l+1}=\frac{1}{2 l(2 l+1)-\mu}$.

Let $l_{0}$ the index from which $\frac{2 l(2 l-1)}{2 l(2 l+1)-\mu} \leq 1$.

Let $u_{l}=\sum_{i=j+2}^{n}\left|S_{i l}\right|=\sum_{i=j+2}^{l}\left|S_{i l}\right|$. For $l \geq l_{0}:$

$$
\begin{aligned}
u_{l+1} & \leq u_{l}+\frac{1}{2 l(2 l+1)-\mu} \leq u_{l_{0}}+\sum_{k=l_{0}}^{l} \frac{1}{2(k-j)(2(k+j)+1)}=u_{l_{0}}+\sum_{q=l_{0}-j}^{l-j} \frac{1}{2 q(2 q+4 j+1)} \\
& \leq u_{l_{0}}+\sum_{k=1}^{\infty} \frac{1}{2 k(2 k+1)} \leq u_{l_{0}}+1-\ln 2 .
\end{aligned}
$$

For $i \leq j+1$,

$$
S_{i l}=T_{i l} \sum_{k=i-1, k \neq j}^{l-1} \frac{1}{2 k(2 k+1)-\mu},
$$


if $l \geq i$ and else 0 .

We deduce that

$$
\begin{aligned}
S_{i l+1} & =T_{i l} \frac{2 l(2 l-1)}{2 l(2 l+1)-\mu}\left(\sum_{k=i-1, k \neq j}^{l-1} \frac{1}{2 k(2 k+1)-\mu}+\frac{1}{2 l(2 l+1)-\mu}\right) \\
& =\frac{2 l(2 l-1)}{2 l(2 l+1)-\mu} S_{i l}+\frac{2 l(2 l-1)}{(2 l(2 l+1)-\mu)^{2}} T_{i l} .
\end{aligned}
$$

For $l \geq l_{0}$ :

$$
\begin{aligned}
\left|S_{i l+1}\right| & \leq\left|S_{i l}\right|+\|T\|^{\infty} \frac{1}{2 l(2 l+1)-\mu} \leq\left|S_{i l_{0}}\right|+\|T\|^{\infty} \sum_{k=l_{0}}^{l} \frac{1}{2 k(2 k+1)-\mu} \\
& \leq\left|S_{i l_{0}}\right|+\|T\|^{\infty}(1-\ln 2) .
\end{aligned}
$$

To evaluate the 1 -norm of the column-vector of index $l$ of $S,\left(l \geq l_{0}\right)$, we add:

$$
\begin{aligned}
\left\|S_{l}\right\|_{1} & =\sum_{i=1}^{j+1}\left|S_{i l}\right|+u_{l} \leq \sum_{i=1}^{j+1}\left|S_{i l_{0}}\right|+u_{l_{0}}+\left((j+1)\|T\|^{\infty}+1\right)(1-\ln (2)) \\
& =\left\|S_{l_{0}}\right\|_{1}+\left((j+1)\|T\|^{\infty}+1\right)(1-\ln (2)) .
\end{aligned}
$$

This proves that there exists an upper bound for $q_{n}$ and $s_{n}$, independent of $n$.

To conclude, we give an upper bound for the coefficients $\alpha_{p}^{(n)}$ of the series $\delta_{2 j}^{(n)}(\tau)$, independent of $n$. To do this, we put $\rho_{n}=\sqrt{\frac{p_{n}}{s_{n}}} \frac{1}{\sqrt{p_{n} s_{n}}+\sqrt{q_{n}}}$ and $r_{n}=\left(\sqrt{p_{n} s_{n}}+\sqrt{q_{n}}\right)^{-2}$. It is proved in [[10], p. 91] that $\rho_{n} \leq d$, the isolation distance of the eigenvalues of $F^{(n)}(d=6$ for all $n)$, and we have seen above that there exists a lower bound for $r_{n}$, say $r$.

Choose for $\Gamma$ the circle $|\zeta-2 j(2 j+1)|=\rho_{n}$. For $|\tau|^{2}<r_{n}$, the series expansion of the resolvant of the perturbated matrix $F^{(n)}+\tau^{2} G^{(n)}, R\left(\zeta, \tau^{2}\right)$, is uniformely convergent for $\zeta \in \Gamma$, and the function $\delta_{2 j}^{(n)}(\tau)-2 j(2 j+1)$ is holomorphic and bounded by $\rho_{n}$ for $|\tau|^{2}<r_{n}$. It follows from Cauchy's inequality for the Taylor coefficients that $\left|\alpha_{p}^{(n)}\right| \leq \rho_{n} r_{n}^{-p} \leq d r^{-p}$. The end of the proof is the same as for theorem 1.

Considering the odd solution $y_{2}$ in the neigborhood of 0 , we introduce an other family of polynomials, defined by:

$$
Q_{0}=0, Q_{1}=2, Q_{2}=0, Q_{3}=\frac{2-\mu}{3}, Q_{4}=0, Q_{5}=\frac{\mu^{2}}{60}+\frac{\tau^{2}}{10}-\frac{7 \mu}{30}+\frac{2}{5},
$$

and the recurrence equation:

$$
-n(n+1) Q_{n}+\left(n^{2}-\mu-n\right) Q_{n-2}+\tau^{2} Q_{n-4}=0 .
$$

The odd coefficients $Q_{2 n+1},(n \geq 0)$ are polynomials of degree $n$ in the variable $\mu$, and we obtain a similar result about the convergence of their roots to the eigenvalues of odd indices of $\mathcal{D}_{\tau}$. 


\section{The eigenvalues and the formal solutions at the point 1}

In the neighborhood of 1 , we know a basis of solutions $f_{1}(x)$ and $f_{2}(x)$, where $f_{1}$ is a convergent series, whose first terms can be computed:

$f_{1}(x)=1+\frac{\mu-\tau^{2}}{2}(x-1)+\left(\frac{1}{16} \tau^{4}-\frac{1}{8} \mu \tau^{2}+\frac{1}{16} \mu^{2}-\frac{1}{8} \tau^{2}-\frac{1}{8} \mu\right)(x-1)^{2}+O\left((x-1)^{3}\right)$

Let $U_{n}$ the coordinates of this series in the basis $(x-1)^{n}$ :

$$
U_{0}=1, U_{1}=\frac{\mu-\tau^{2}}{2}, U_{2}=\frac{1}{16} \tau^{4}-\frac{1}{8} \mu \tau^{2}+\frac{1}{16} \mu^{2}-\frac{1}{8} \tau^{2}-\frac{1}{8} \mu,
$$

the following polynomials satisfying the recurrence relation:

$$
2 n^{2} U_{n}+\left(n^{2}+\tau^{2}-\mu-n\right) U_{n-1}+2 \tau^{2} U_{n-2}+\tau^{2} U_{n-3}=0 .
$$

Proposition 11. The polynomials $U_{n}$ are polynomials of degree $n$ in the variable $\mu$ and for all $n \geq 1, U_{n} \bmod \tau^{2}=\frac{1}{2^{n}(2 n) !^{2}} \prod_{j=0}^{n-1}(\mu-j(j+1))$. The polynomial $U_{n}$ admits $n$ Puiseux series solutions, we will note $\gamma_{j}^{(n)}, j=0 \ldots n-1$, the series whose constant term is $j(j+1)$.

Proof: Just reduce the recurrence relation modulo $\tau^{2}$ :

$$
2 n^{2}\left(U_{n} \bmod \tau^{2}\right)=(\mu-n(n-1))\left(U_{n-1} \bmod \tau^{2}\right) .
$$

Proposition 12. For all $j \geq 0$, the series $\hat{\mu}_{2 j}$ satisfies:

$$
\forall n>j, U_{2 n-1}\left(\hat{\mu}_{2 j}\right)=0 \quad \bmod \tau^{2(n-j)} \quad \text { and } \quad U_{2 n}\left(\hat{\mu}_{2 j}\right)=0 \quad \bmod \tau^{2(n-j)} .
$$

For the proof of this proposition, we will need the

Lemma 2. For all $j \geq 0$,

$$
\sum_{i=0}^{\infty} P_{2 i}(2 j(2 j+1)) \quad \bmod \tau^{2}=\sum_{i=0}^{2 j} P_{2 i}\left(2 j(2 j+1) \quad \bmod \tau^{2}=\frac{(-1)^{j} 4^{j}(j !)^{2}}{(2 j) !} \bmod \tau^{2} .\right.
$$

Proof: For $j=0$, we verify $P_{0}(0)=1$ and $P_{2 i}(0)=0 \bmod \tau^{2}$.

For $j \geq 1$, the first equality comes from the fact that $P_{2 i}\left(2 j(2 j+1)=0 \bmod \tau^{2}\right.$, for all $i>j$.

The second equality can be established by applying the divided differences, in order to compute the coordinates in the Newton basis $\left(1, \mu-x_{0},\left(\mu-x_{0}\right)\left(\mu-x_{1}\right), \ldots, \prod_{i=0}^{j}\left(\mu-x_{i}\right)\right)$ of the polynomial of degree less or equal $j$ interpolating the points $\left(x_{0}, z_{0}\right),\left(x_{1}, z_{1}\right), \ldots,\left(x_{j}, z_{j}\right)$. From the values $z_{i}$, we build a lower triangular array in the following way: for $i=0 \ldots j$, $C[i, 0]=z_{i}$ (the first column), then for $i=1 \ldots j, C[i, 1]=\frac{C[i, 0]-C[i-1,0]}{x_{i}-x_{i-1}}$ (the second column), for $i=k \ldots j, C[i, k]=\frac{C[i, k-1]-C[i-1, k-1]}{x_{i}-x_{i-k}}$, at last $C[j, j]=\frac{C[j, j-1]-C[j-1, j-1]}{x_{j}-x_{0}}$. It is 
well known that the coordinates in the Newton basis of the interpolation polynomial are the diagonal elements $C[i, i]$.

In our case, we are knowing the coordinates in the Newton basis, and we want the value of the polynomial at the points $x_{i}$. Indeed $x_{i}=2 i(2 i+1)$ and $C[i, i]=\frac{(-1)^{i}}{(2 i) !}$. We deduce an exact formula for all the elements of the array:

$$
\forall k \geq i, C[i, k]=(-1)^{i} 4^{i-k} \frac{i !(i-k) !}{k !(2 i) !} .
$$

We prove this formula by recurrence on the index $i$. For $i=0, C[0,0]=1$.

Assume that the formula has been proved for the row $i$. We prove it for the row $i+1$, starting from the known coefficient $C[i+1, i+1]=\frac{(-1)^{i+1}}{(2 i+2) !}$. Assuming that $C[i+1, k]$ and $C[i, k]$ are known, we compute $C[i+1, k-1]=\left(x_{i+1}-x_{i+1-k}\right) C[i+1, k]+C[i, k-1]$ and we find $C[i+1, k-1]=(-1)^{i+1} 4^{i+2-k} \frac{(i+1) !(i+2-k) !}{(k-1) !(2 i+2) !}$.

Conclusion: $C[j, 0]=(-1)^{j} 4^{j} \frac{j ! j !}{(2 j) !}$.

Proof: Consider again the eigenfunction $y_{1}(x)$, corresponding to an eigenvalue of even index. It is proportional to $f_{1}(x)$, which means $y_{1}(x)=\mathbf{c} f_{1}(x)$.

Then we obtain by change of basis:

$$
\left(\begin{array}{c}
\mathbf{c} \\
\mathbf{c} U_{1} \\
\mathbf{c} U_{2} \\
\mathbf{c} U_{3} \\
\mathbf{c} U_{4} \\
\vdots
\end{array}\right)=B\left(\begin{array}{c}
1 \\
0 \\
P_{2} \\
0 \\
P_{4} \\
\vdots
\end{array}\right)
$$

where we just know that $B$ is an infinite upper triangular matrix, whose elements will be noted $\beta_{i j}$.

$$
B=\left(\begin{array}{ccccc}
1 & 1 & 1 & 1 & \ldots \\
& 1 & 2 & 3 & \ldots \\
& & 1 & 3 & \ldots \\
& & & 1 & \ldots \\
& & & & \ddots
\end{array}\right)
$$


Then: $\mathbf{c}=\sum_{i=0} P_{2 i}$ and, applying the proposition $9, \mathbf{c}\left(\hat{\mu}_{0}\right)=1 \bmod \tau^{2}$. We deduce:

$$
\begin{gathered}
\forall i \geq 1, \mathbf{c}\left(\hat{\mu}_{0}\right) U_{2 i-1}\left(\hat{\mu}_{0}\right)=\beta_{2 i-1,2 i} P_{2 i}\left(\hat{\mu}_{0}\right) \quad \bmod \tau^{2 i+2}=0 \bmod \tau^{2 i} \\
\mathbf{c}\left(\hat{\mu}_{0}\right) U_{2 i}\left(\hat{\mu}_{0}\right)=\beta_{2 i, 2 i} P_{2 i}\left(\hat{\mu}_{0}\right) \quad \bmod \tau^{2 i+2}=0 \quad \bmod \tau^{2 i}
\end{gathered}
$$

Also $\mathbf{c}\left(\hat{\mu}_{2}\right)=1+P_{2}\left(\hat{\mu}_{2}\right) \bmod \tau^{2} \neq 0 \bmod \tau$.

We deduce: $\mathbf{c}\left(\hat{\mu}_{2}\right) U_{3}\left(\hat{\mu}_{2}\right)=4 P_{4}\left(\hat{\mu}_{2}\right) \bmod \tau^{4}=0 \bmod \tau^{2}$, thus $U_{3}\left(\hat{\mu}_{2}\right)=0 \bmod \tau^{2}$. And $\mathbf{c}\left(\hat{\mu}_{2}\right) U_{4}\left(\hat{\mu}_{2}\right)=P_{4}\left(\hat{\mu}_{2}\right) \bmod \tau^{4}=0 \bmod \tau^{2}$, thus $U_{4}\left(\hat{\mu}_{2}\right)=0 \bmod \tau^{2}$.

In a general way

$$
\mathbf{c}\left(\hat{\mu}_{2 j}\right)=\sum_{i=0}^{\infty} \beta_{1,2 j} P_{2 i}\left(\hat{\mu}_{2 j}\right)=\sum_{i=0}^{2 j} P_{2 i}\left(\hat{\mu}_{2 j}\right) \quad \bmod \tau^{2} \neq 0 \quad \bmod \tau .
$$

The last inequality is a consequence of lemma 2 .

We deduce:

$$
\forall n>j, \mathbf{c}\left(\hat{\mu}_{j}\right) U_{2 n-1}\left(\hat{\mu}_{2 j}\right)=\beta_{2 n-1, n} P_{2 n}\left(\hat{\mu}_{j}\right) \quad \bmod \tau^{2(n+1-j)}=0 \bmod \tau^{2(n-j)},
$$

thus $U_{2 n-1}\left(\hat{\mu}_{2 j}\right)=0 \bmod \tau^{2(n-j)}, \forall n>j$.

And

$$
\mathbf{c}\left(\hat{\mu}_{j}\right) U_{2 n}\left(\hat{\mu}_{2 j}\right)=\beta_{2 n, n} P_{2 n}\left(\hat{\mu}_{j}\right) \bmod \tau^{2(n+1-j)}=0 \bmod \tau^{2(n-j)},
$$

thus $U_{2 n}\left(\hat{\mu}_{2 j}\right)=0 \bmod \tau^{2(n-j)}, \forall n>j$.

Proposition 13. Link between the series $\gamma_{2 j}^{(n)}$ and the series $\hat{\mu}_{2 j}$

$$
\forall j \geq 0, \forall n>j, \quad \gamma_{2 j}^{(2 n-1)}=\gamma_{2 j}^{(2 n)}=\hat{\mu}_{2 j} \quad \bmod \tau^{2(n-j)} .
$$

THEOREM 3. For $\tau$ small enough, the sequence $\gamma_{2 j}^{(n)}(\tau)$ converges to $\hat{\mu}_{2 j}(\tau)$, when $n$ tends to infinity.

Proof: If we try to make exactly the same work on the recurrence relation satisfied by $U_{n}$, it is easy to compute new matrices $F^{(n)}, G^{(n)}, T^{(n)}, S^{(n)}$, but the 1-norm of $G^{(n)} T^{(n)}$ is not bounded.

We put $\mathbb{U}_{n}=2^{n} U_{n}$. Of course $\mathbb{U}_{n}$ has the same roots as $U_{n}$ and satisfies the recurrence relation:

$$
n^{2} \mathbb{U}_{n}+\left(n^{2}+\tau^{2}-\mu-n\right) \mathbb{U}_{n-1}+4 \tau^{2} \mathbb{U}_{n-2}+4 \tau^{2} \mathbb{U}_{n-3}=0 .
$$


Thus $\mathbb{U}_{n+1}(\mu)=0$ if and only if

$$
\left(\begin{array}{cccccccc}
\tau^{2}-\mu & 1 & & & & & & \\
4 \tau^{2} & \tau^{2}-\mu+2 & 4 & & & & & \\
4 \tau^{2} & 4 \tau^{2} & \tau^{2}-\mu+6 & 9 & & & \\
& \ddots & \ddots & \ddots & \ddots & & & \\
& & 4 \tau^{2} & 4 \tau^{2} & \tau^{2}-\mu+k(k+1) & (k+1)^{2} & & \\
& & & \ddots & \ddots & \ddots & \ddots & \\
& & & & 4 \tau^{2} & 4 \tau^{2} & \tau^{2}-\mu+n(n-1) & n^{2} \\
& & & & & 4 \tau^{2} & 4 \tau^{2} & \tau^{2}-\mu+n(n+1)
\end{array}\right)\left(\begin{array}{c}
\mathbb{U}_{0} \\
\mathbb{U}_{1} \\
\vdots \\
\mathbb{U}_{k} \\
\vdots \\
\mathbb{U}_{n-1} \\
\mathbb{U}_{n}
\end{array}\right)=0 .
$$

We are led to search the eigenvalues of the perturbated matrix:

$$
F^{(n)}+\tau^{2} G^{(n)},
$$

where

$$
F^{(n)}=\left(\begin{array}{ccccc}
0 & 1 & & & \\
& 2 & 4 & & \\
& \ddots & \ddots & \\
& & (n-1) n & n^{2} \\
& & & n(n+1)
\end{array}\right), \quad G^{(n)}=\left(\begin{array}{ccccc}
1 & & & & \\
4 & 1 & & & \\
4 & 4 & 1 & & \\
& \ddots & \ddots & \ddots & \\
& & 4 & 4 & 1
\end{array}\right) .
$$

Here again the resolvant $R^{(n)}(\zeta)$ is an upper triangular matrix whose elements can be computed explicitely (by omitting the upper index $(n)$ ):

$$
R_{i i}(\zeta)=-1, \quad \text { and } R_{i l}(\zeta)=-\frac{\prod_{k=i-1}^{l-2}(k+1)^{2}}{\prod_{k=i-1}^{l-1}(\zeta-k(k+1))}, \text { if } l>i,
$$

and we can expand these elements in the neighborhood of the eigenvalue $\mu=j(j+1)$. The first $j$ columns and the rows of index greater than $j+1$ of the matrix $T^{(n)}$ are null. For $i \leq j+1, T_{i i}=-1$ and if $l>j, T_{i l}=-R_{i l}(\zeta) \times(\zeta-\mu)$ evaluated in $\mu$. Therefore

$$
T_{i l}=\frac{\prod_{k=i-1}^{l-2}(k+1)^{2}}{\prod_{k=i-1, k \neq j}^{l-1}(\zeta-k(k+1))} .
$$

Thus $T_{i l+1}=\frac{-l^{2}}{l(l+1)-\mu} T_{i l}$. For all $j$, there exists an index $l_{0}$ from which the ratio $\frac{l^{2}}{l(l+1)-\mu}$ is lower than 1 ; from this index, the 1 -norm of the columns of $T^{(n)}$ are de- 
creasing. To conclude, $\left\|G^{(n)} T^{(n)}\right\|_{1} \leq\left\|G^{(n)}\right\|_{1}\left\|T^{(n)}\right\|_{1}=9\left\|T^{(n)}\right\|_{1}$, and there exists an upper bound of $p_{n}$ independent of $n$.

The matrix $S$ has the same structure as in the previous paragraph.

For $i>j+1, \mu$ is not a pole, thus $S_{i l}=R_{i l}(\mu)$, that means:

$$
S_{i l}=\frac{\prod_{k=i-1}^{l-2}(k+1)^{2}}{\prod_{k=i-1}^{l-1}(\mu-k(k+1))}
$$

if $l \geq i$ and else 0 .

As above, $T_{i l+1}=\frac{l^{2}}{l(l+1)-\mu} S_{i l}$ if $l \geq i, S_{l+1 l+1}=\frac{1}{l(l+1)-\mu}$.

Let $l_{0}$ be the index from which $\frac{l^{2}}{l(l+1)-\mu} \leq 1$.

Let $u_{l}=\sum_{i=j+2}^{n}\left|S_{i l}\right|=\sum_{i=j+2}^{l}\left|S_{i l}\right|$. For $l \geq l_{0}$ :

$$
\begin{aligned}
u_{l+1} & \leq u_{l}+\frac{1}{l(l+1)-\mu} \leq u_{l_{0}}+\sum_{k=l_{0}}^{l} \frac{1}{(k-j)(k+j+1)}=u_{l_{0}}+\sum_{q=l_{0}-j}^{l-j} \frac{1}{q(q+2 j+1)} \\
& \leq u_{l_{0}}+\sum_{k=1}^{\infty} \frac{1}{k(k+1)} \leq u_{l_{0}}+1
\end{aligned}
$$

For $i \leq j+1$,

$$
S_{i l}=T_{i l} \sum_{k=i-1, k \neq j}^{l-1} \frac{1}{k(k+1)-\mu}
$$

if $l \geq i$ and else 0 .

We deduce

$$
\begin{aligned}
S_{i l+1} & =T_{i l} \frac{l^{2}}{l(l+1)-\mu}\left(\sum_{k=i-1, k \neq j}^{l-1} \frac{1}{k(k+1)-\mu}+\frac{1}{l(l+1)-\mu}\right) \\
& =\frac{l^{2}}{l(l+1)-\mu} S_{i l}+\frac{l^{2}}{(l(l+1)-\mu)^{2}} T_{i l} .
\end{aligned}
$$

For $l \geq l_{0}$ :

$$
\left|S_{i l+1}\right| \leq\left|S_{i l}\right|+\|T\|^{\infty} \frac{1}{l(l+1)-\mu} \leq\left|S_{i l_{0}}\right|+\|T\|^{\infty} \sum_{k=l_{0}}^{l} \frac{1}{k(k+1)-\mu} \leq\left|S_{i l_{0}}\right|+\|T\|^{\infty} .
$$


To compute the 1 -norm of the column vector of index $l$ of $S,\left(l \geq l_{0}\right)$, we add:

$$
\left\|S_{l}\right\|_{1}=\sum_{i=1}^{j+1}\left|S_{i l}\right|+u_{l} \leq \sum_{i=1}^{j+1}\left|S_{i l_{0}}\right|+u_{l_{0}}+(j+1)\|T\|^{\infty}+1=\left\|S_{l_{0}}\right\|_{1}+(j+1)\|T\|^{\infty}+1 .
$$

This proves that there exists an upper bound for $q_{n}$ and $s_{n}$, independent of $n$.

The end of the proof is the same as for theorem 2 .

\section{The eigenvalues and the formal solutions at infinity}

In the neighborhood of infinity, we recall the formal solutions $\hat{y}_{1}(x)$ and $\hat{y}_{2}(x)$ and we note $V_{n}$ the coordinates of the series $x e^{i \tau x} \hat{y}_{1}(x)$ in the basis $1 / x^{n}$.

$$
V_{0}=1, V_{1}=\frac{-i\left(\mu-\tau^{2}\right)}{2 \tau}, V_{2}=\frac{-\mu^{2}+2 \mu+2 \tau^{2} \mu+2 \tau^{2}-\tau^{4}}{8 \tau^{2}},
$$

the following coefficients satisfying the recurrence relation:

$$
2 n i \tau V_{n}+\left(n^{2}+\tau^{2}-\mu-n\right) V_{n-1}-2 i \tau(n-1) V_{n-2}-(n-1)(n-2) V_{n-3}=0 .
$$

Proposition 14.

$$
\forall n \geq 0, \quad V_{n}=n !\left(\frac{-i}{\tau}\right)^{n} U_{n}
$$

Proof: This is true for $n=0, n=1, n=2$. Assume that the property is true for indices $\leq n-1$. Then

$$
2 n i \tau V_{n}=-\left(n^{2}+\tau^{2}-\mu-n\right) V_{n-1}+2 i \tau(n-1) V_{n-2}+(n-1)(n-2) V_{n-3},
$$

we replace the polynomials $V_{n-1}, V_{n-2}, V_{n-3}$ by their expression in terms of $U_{n-1}, U_{n-2}, U_{n-3}$ and we use the recurrence relation satsified by the polynomials $U_{k}$ to prove that the property is still true for the index $n$.

TheOREM 4. For $\tau$ small enough, the sequence of the zeros of index $2 j$ of $V_{n}$ converges to $\hat{\mu}_{2 j}(\tau)$, when $n$ tends to infinity.

Comment: the result of the proposition 14 is linked to the relations that could been obtained by using Fourier transform. In particular, the operator $\mathcal{D}_{1}$ is "invariant by Fourier transform" (cf. [12], 3.3, page 356) and for $\tau=1$, the series $f_{1}$, interpreted as a power series in the variable $(x-1)$ is the Borel-transform of the series $-i e^{i x} \hat{y}_{1}$, interpreted as a power series in the variable $\frac{-i}{x}$. Neverthless, we derive here in a very simple way the relation between the polynomials $U_{n}$ and the coefficients $V_{n}$, which is suffisant for our initial goal: prove that the zeros of $V_{n}$ converge to the eigenvalues of the differential operator $\mathcal{D}_{\tau}$ (for small $\tau$ ). 


\section{Asymptotic behavior of the coefficients $\mathbb{P}_{n}(\mu)$}

The study of the asymptotic behavior of $\mathbb{P}_{n}(\mu)$, defined by (3), based on [13], will enable us to prove that $\hat{\mu}_{2 j}(\tau)$ (if it converges) is an eigenvalue of $\mathcal{D}_{\tau}$.

The recurrence equation satisfied by the polynomials $\mathbb{P}_{n}$,

$$
\left(1+\frac{1}{2 n}\right)\left(1+\frac{1}{n}\right) \mathbb{P}_{n+1}(\mu)-\left(1+\frac{1}{2 n}-\frac{\mu}{4 n^{2}}\right) \mathbb{P}_{n}(\mu)-\frac{\tau^{2}}{4 n^{2}} \mathbb{P}_{n-1}(\mu)=0,
$$

is irregular and its characteristic equation is: $z^{2}-z-\frac{\tau^{2}}{4 n^{2}}=0$. We search the leading terms of the Puiseux solutions, that means a beginning of solution of the form $z=\rho n^{s}$ and find two possibilities: $s=0, z=1$, and $s=-2, z=\frac{-\tau^{2}}{4}$.

Each "solution" $\rho n^{s}$ corresponds to an asymptot of the recurrence equation of the form $\rho^{n} \Gamma(n)^{s} p_{n}$, and we have to complete the determination of $p_{n}$.

- $s=-2$. Plugging $\mathbb{P}_{n}=\left(\frac{-\tau^{2}}{4}\right)^{n} \Gamma(n)^{-2} p_{n}$ in the initial recurrence, we obtain for $p_{n}$ :

$$
-\frac{\tau^{2}}{4 n^{2}}\left(1+\frac{1}{2 n}\right)\left(1+\frac{1}{n}\right) p_{n+1}-\left(1+\frac{1}{2 n}-\frac{\mu}{4 n^{2}}\right) p_{n}+\left(1-\frac{1}{n}\right)^{2} p_{n-1}=0 .
$$

Then $p_{n} \approx n^{-5 / 2}$. And so we have the first asymptotic behavior:

$C_{1}=\left(\frac{-\tau^{2}}{4}\right)^{n} \Gamma(n)^{-2} n^{-5 / 2}$

- $s=0$.

$$
\left(1+\frac{1}{2 n}\right)\left(1+\frac{1}{n}\right) p_{n+1}-\left(1+\frac{1}{2 n}-\frac{\mu}{4 n^{2}}\right) p_{n}-\frac{\tau^{2}}{4 n^{2}} p_{n-1}=0 .
$$

Then: $p_{n} \approx \frac{1}{n}$. The second behavior is: $C_{2}=\frac{1}{n}$.

Then $\mathbb{P}_{n}(\mu) \approx a(\mu) C_{1}+b(\mu) C_{2}$.

And $\mu$ is an eigenvalue if and only if $\sqrt[2 n]{\left|\mathbb{P}_{n}(\mu)\right|}$ tends to 0 .

Now $\sqrt[2 n]{\left|C_{1}\right|} \approx \frac{\tau e}{2 n} ; \sqrt[2 n]{C_{2}} \approx 1$

Thus $\mu$ is an eigenvalue if and only if $b(\mu)=0$. Finally, if $\mu$ is not an eigenvalue, $b(\mu) \neq 0$, thus $\mathbb{P}_{n}(\mu)$ tends to 0 as $\frac{b(\mu)}{n}$.

Proposition 15. Assume that a sequence $\mu_{n}$ is known, which converges to $\mu$, and satisfies $\mathbb{P}_{n}\left(\mu_{n}\right)=0$, for all $n$. Then $\mu$ is an eigenvalue of $\mathcal{D}_{\tau}$.

Proof: Assume that $\mu$ is not an eigenvalue. Then $\mathbb{P}_{n}(\mu) \approx \frac{b(\mu)}{n}$. The polynomials $\mathbb{P}_{n}^{\prime}$ satisfy the recurrence relation:

$$
\tau^{2} \mathbb{P}_{n-1}^{\prime}+(2 n(2 n+1)-\mu) \mathbb{P}_{n}^{\prime}-(2 n+1)(2 n+2) \mathbb{P}_{n+1}^{\prime}=\mathbb{P}_{n} .
$$


We deduce that the asymptotic behavior of $\mathbb{P}_{n}^{\prime}(\mu)$ is at worst $\frac{c s t}{n}$. Then:

$$
\left|\mathbb{P}_{n}(\mu)\right|=\left|\mathbb{P}_{n}(\mu)-\mathbb{P}_{n}\left(\mu_{n}\right)\right| \leq \frac{c s t}{n}\left|\mu-\mu_{n}\right|,
$$

which is in contradiction with the asyptotic behavior of $\left|\mathbb{P}_{n}(\mu)\right|$.

Conclusion: $\mu$ is an eigenvalue of $\mathcal{D}_{\tau}$.

Corollary 2: for $\tau$ small enough, $\hat{\mu}_{2 j}(\tau)$ is an eigenvalue of $\mathcal{D}_{\tau}$.

The following corollary and its variants summarize some of our main results. (We denote $\delta_{\eta}$ the Dirac mass at $\eta \in \mathbb{R}$.)

Corollary 3: For $\tau \geq 0$, we denote by $\left\{\mu_{r}(\tau)\right\}_{r \in \mathbb{N}}$ the set of eigenvalues of $\mathcal{D}_{\tau}$ and, for all $n \in \mathbb{N}$, by $\left\{\nu_{p}(\tau)\right\}_{p=0, \ldots, n-1}$ the set of roots of the polynomial $P_{2 n}{ }^{6}$ (as a polynomial in $\mu$, $\tau$ being fixed).

There exists $\rho>0$ such that if $0 \leq \tau<\rho$, is fixed, then, for all $n \in \mathbb{N}$, the roots $\left\{\nu_{p}(\tau)\right\}_{p=0, \ldots, n-1}$ are real and distinct and such that the measure $\sum_{p=0, \ldots, n-1} \delta_{\nu_{p}(\tau)}$ tends to the measure $\sum_{p \in \mathbb{N}} \delta_{\mu_{2 p}(\tau)}$ when $n$ tends to $+\infty$.

There are similar results (mutatis mutandis) replacing the sequence of polynomials $P_{2 n}$ by the sequences $Q_{2 n+1}, U_{n}, V_{n}$ and $D_{n}$.

\section{Asymptotic expansions of the eigenvalues for large values of $\tau$}

In order to study the eigenvalues of $\mathcal{D}_{\tau}$ for "big values" of $\tau$ one can use, as for "small values" of $\tau$, a perturbative method. The idea is to set $x:=\xi / \sqrt{2 \tau}$ (cf. $[14,3])$. Then $\mathcal{D}_{\tau} y-\mu y=0$ is transformed into:

$$
\left(\xi^{2}-2 \tau\right) \frac{d^{2} y}{d \xi^{2}}+2 \xi \frac{d y}{d \xi}+\left(\frac{\tau \xi^{2}}{2}-\mu\right) y
$$

or equivalently (for $\tau>0$ ) into:

$$
\frac{d^{2} y}{d \xi^{2}}+\left(\frac{\mu}{2 \tau}-\frac{\xi^{2}}{4}\right) y-\frac{\xi^{2}}{2 \tau} \frac{d^{2} y}{d \xi^{2}}-\frac{\xi}{\tau} \frac{d y}{d \xi}
$$

which we can interpret, when $\tau$ is "large", as a perturbation of

$$
\frac{d^{2} y}{d \xi^{2}}+\left(\frac{\mu}{2 \tau}-\frac{\xi^{2}}{4}\right) y
$$

This differential equation is a particular case of the parabolic-cylinder differential equation:

$$
\frac{d^{2} y}{d \xi^{2}}+\left(r+\frac{1}{2}-\frac{\xi^{2}}{4}\right) y
$$

\footnotetext{
${ }^{6}$ The roots are complex numbers and if necessary they are repeated according to their multiplicity.
} 
For $r \in \mathbb{N}$, this equation admits as a solution:

$$
\mathbb{D}_{r}(\xi)=(-1)^{r} e^{\xi^{2} / 4} \frac{d^{r}}{d \xi^{r}} e^{-\xi^{2} / 2}=2^{-r / 2} e^{-\xi^{2} / 4} H_{r}(\xi / \sqrt{2}),
$$

where $H_{r}$ is a Hermite polynomial.

The foregoing suggests that we expand $y$ in terms of the parabolic cylinder functions:

$$
y=\sum_{n=0}^{+\infty} h_{n} \mathbb{D}_{n} .
$$

The coefficients are solutions of a five terms linear recurrence (cf. [3]). From this recurrence one obtains asymptotic expansions of the eigenvalues $\mu_{n}$ in the variable $\tau^{-1}$ (cf. [2] 3.25 Satz 9, page 243, [15] 21.7.6 page 321, [3] (8.1.11) page 60):

$$
\mu_{n}=(2 n+1) \tau-\left(2 n^{2}+2 n+3\right) 2^{-2}-(2 n+1)\left(n^{2}+n+3\right) 2^{-4} \tau^{-1}+\cdots
$$

Then it is natural to try to imitate what we did above in order to get similar results for large values of $\tau^{7}$, replacing the expansion:

$$
y(x)=\sum_{n=0}^{\infty} a_{n} L_{n}(x) .
$$

by the expansion:

$$
y(x)=\sum_{n=0}^{+\infty} h_{n} \mathbb{D}_{n}(x),
$$

$\tau$ by $\tau^{-1}, \mu$ by $\mu / \tau$ and the polynomials $P(\tau, \mu)$ in $(\tau, \mu)$, of degree $n$ in $\mu$, by $Q\left(\tau^{-1}, \mu \tau^{-1}\right):=$ $P(\tau, \mu) \tau^{-n}$. gent.

Unfortunately this idea does not work because the power series expansions (8) are diver-

The divergence was proved in [2], 3.253, page 247. The authors compute a power series expansion of $\mu_{n}(\tau)$ for purely imaginary values of $\tau\left(\tau=i \tau^{*}, \tau^{*}>0\right)$ and remark that it does not match with the expansion (8).

It is interesting to compare to what it is happening with the anharmonic oscillator whose potential is $V(x):=x^{2}+\beta x^{4}$ (a triconfluent Heun equation). The eigenvalues $\lambda_{n}\left(n \in \mathbb{N}^{*}\right)$ are real and real analytic functions defined on the positive ray. In 1969 Bender and Wu [16] studied analytic continuation of $\lambda_{n}$ to the $\beta$-plane. They discovered that:

- for all $m, n \in \mathbb{N}^{*}, \lambda_{m}$ is an analytic continuation of $\lambda_{n}$;

- the singularities encountered in the analytic continuation of the eigenvalues are algebraic ramification points accumulating to $\beta=0$;

- the formal power series expansions of $\lambda_{n}$ in powers of $\beta$ are divergent.

\footnotetext{
${ }^{7}$ There is a strong numerical evidence for such results.
} 
The proofs of these results were completed later by various authors (in particular LoeffelMartin, B. Simon, Eremenko-Gabrielov). There are also a very interesting attempt to obtain such results using the Ecalle resurgence theory (based on Borel transformation) [17]. Unfortunately some proofs remain uncomplete.

By analogy (replacing $\beta$ by $\tau^{-1}$ ) it would be interesting to try to apply resurgence methods to the study of the eigenvalues of the prolate spheroidal problems. The first test in this direction is the following conjecture.

Conjecture 1: The divergent power series expansion (8) (in $\tau^{-1}$ ) is Gevrey of order 1.

In a recent work [18] G. Başar and G. V. Dunne investigated the resurgence of the asymptotic expansions of the eigenvalues in the cases of Mathieu and Lamé equations in relation with some problems of gauge theory in physics.

As for the prolate spheroidal case it is also possible in the case of the anharmonic oscillator to get convergent expansions of the eigenvalues. The idea is to use a change of variables due to Symanzik to "replace" the potential $V(x):=x^{2}+\beta x^{4}$ by the potential $V(x):=x^{4}+\alpha x^{2}$. Then the power expansions in $\alpha$ are convergent (cf. [19]) as the power expansions in $\tau$ in the prolate spheroidal case.

\section{Stokes phenomena and analytic continuation of the eigenvalues in the complex $\tau$ plane}

In this part we shall consider the operator:

$$
\mathcal{D}_{\tau}=\left(x^{2}-1\right) \frac{d}{d x}^{2}+2 x \frac{d}{d x}+\tau^{2} x^{2}
$$

when $\tau$ is a complex parameter. Setting $\tau:=i \tau^{*}$, we get $\tau^{2}=-\left(\tau^{*}\right)^{2}$ and $\mathcal{D}_{\tau}=\left(x^{2}-1\right) \frac{d^{2}}{d x}+$ $2 x \frac{d}{d x}-\left(\tau^{*}\right)^{2} x^{2}$, therefore we get also the case of the oblate spheroidal functions of order 0 .

Definition 1. If $\tau \in \mathbb{C}$, then an eigenvalue of $\mathcal{D}_{\tau}$ is by definition a complex number $\mu \in \mathbb{C}$ such that there exists a non trivial analytic solution at 0 of:

$$
\mathcal{D}_{\tau}(y)=\mu y
$$

such that its analytic continuation is bounded on $]-1,1[$. Then such an analytic function is called an eigenfunction of $\mathcal{D}_{\tau}$ associated to $\mu$.

This definition is clearly equivalent to the classical definition when $\tau$ is real. The eigenvalues in the complex case have been studied by various authors (cf. in particular [14]).

Remark. Let $\tau:=|\tau| e^{i \theta} \in \mathbb{C}$, then if $\mu \in \mathbb{C}$ is an eigenvalue of $\mathcal{D}_{\tau}$, the corresponding solution at 0 , bounded on ] $-1,1$ [ extends in an entire function $y$ such that $y(z)$ tends to 0 as $z \rightarrow \infty$ along the rays of argument $\pm \theta$. Therefore our definition of eigenvalues is in accordance with definitions of other authors in the study of the Schrödinger equation in the complex domain (cf. [21]). 
In [1] we studied the Stokes phenomena for $\mathcal{D}_{\tau}$ when $\tau$ is real. Our study extends easily to the complex case $\tau:=|\tau| e^{i \theta} \in \mathbb{C}$. Using an evident generalisation of the notations of [1] we get two Stokes matrices (respectively associated to the two Stokes rays $\theta \pm \pi / 2$ ):

$$
S^{\theta+\pi / 2}:=\left(\begin{array}{ll}
1 & \alpha \\
0 & 1
\end{array}\right) \quad \text { and } S^{\theta-\pi / 2}:=\left(\begin{array}{cc}
1 & 0 \\
\beta & 1
\end{array}\right) .
$$

We have $\operatorname{Tr}\left(S^{\theta-\pi / 2} S^{\theta+\pi / 2}\right)=2+\alpha \beta$ therefore $\alpha \beta$ is well defined and is an entire function of $(\tau, \mu) \in \mathbb{C}^{2}$. We define $F:(\tau, \mu) \in \mathbb{C}^{2} \mapsto \alpha(\tau, \mu) \beta(\tau, \mu)$. By analogy with the case of the quartic oscillator (cf. $[19,20]$ ), we call the entire function $F$ the spectral determinant ${ }^{8}$. We denote by $\mathcal{Z} \subset \mathbb{C}$ the set of zeros of $F: \mathcal{Z}:=\left\{(\tau, \mu) \in \mathbb{C}^{2} \mid F(\tau, \mu)=0\right\}$. It is a plane analytic curve.

TheOREM 5. Let $\tau, \mu \in \mathbb{C}$. the following conditions are equivalent:

(i) $\mu$ is an eigenvalue of $\mathcal{D}_{\tau}$;

(ii) the sums of the power series $f_{1}$ and $g_{1}$ (the analytic solutions at 1 , respectively -1 ) are entire functions;

(iii) the Stokes phenomenon is trivial (i. e. $\alpha=\beta=0$ );

(iv) the monodromy around $[-1,1]$ is trivial.

Moreover if $\mu$ is an eigenvalue of $\mathcal{D}_{\tau}$, then a corresponding eigenfunction is even or odd and the corresponding eigenspace is a complex vector space of dimension 1.

Proof: The proof is a variant of the proof of the theorem 1 of [1]. We will only detail the differences.

Let $\tau, \mu \in \mathbb{C}$. In the neighborhood of the point 1 , we find a basis of solutions $(x \in \mathbb{C})$ of $\mathcal{D}_{\tau}(f)=\mu f$ :

$$
f_{1}(x), \quad f_{1}(x) \log (x-1)+\varphi_{1}(x) .
$$

In the neighborhood of the point -1 , we find a basis of solutions $(x \in \mathbb{C})$ :

$$
g_{1}(x), \quad g_{1}(x) \log (x+1)+\psi_{1}(x) .
$$

All the functions $f_{1}, \varphi_{1}, g_{1}, \psi_{1}$ are sums of convergent power series (respectively in the variables $x-1$ and $x+1$ ), with a radius of convergence at least 2 .

The dimension of the space of solutions holomorphic at 1 (resp. -1 ) is 1 . It is generated by $f_{1}$ (resp. $\left.g_{1}\right)$.

- The properties (iii) and (iv) are equivalent (cf. [1]).

- The property (iv) implies the property (ii) (cf. [1]).

\footnotetext{
${ }^{8}$ In the case of the quartic oscillator and, more generally, in the case of Sibuya's differential equations, the spectral determinant is also defined using Stokes phenomena [21]. In the case of the spheroidal differential equations this approach seems new.
} 
- The property (ii) implies clearly the property (i). We will show that the property (i) implies the property (iv). That will end the proof.

We suppose that $f$ is an eigenfunction of $\mathcal{D}_{\tau}$ associated to $\mu$. Then it extends analytically in a neighborhood of -1 and a neighborhood of 1 . Therefore $f$ extends analytically in an entire function.

The operator $\mathcal{D}_{\tau}$ is invariant under the transformation $x \mapsto-x$, therefore $g: x \mapsto f(-x)$ is also an eigenfunction. Hence $f+g$ and $f-g$ are equal to zero or are eigenfunction. We cannot have $f+g=f-g=0(f \neq 0)$, then there exists an even or an odd eigenfunction.

We suppose that $f$ is even (the odd case is similar). We prove, as in [1], that there exists another independent solution $h:=f \log \frac{x+1}{x-1}+\eta$ where $\eta$ is an entire function. The action of the monodromy around $[-1,1]$ on $f, \eta, \log \frac{x+1}{x-1}$ is trivial and the result follows.

Corollary 4: If $\tau \in \mathbb{C}$, then $\mu \in \mathbb{C}$ is an eigenvalue of $\mathcal{D}_{\tau}$ if and only if $F(\tau, \mu)=0$, that is if $(\tau, \mu) \in \mathcal{Z}$.

THEOREM 6. (i) All the analytic functions $\mu_{p}: \tau \mapsto \mu_{p}(\tau),|\tau|$ "small", are restrictions of two multi-valued ramified analytic functions $\Lambda^{i}, i=0,1$, of $\tau$, one for even $p$ the other for odd $p$. Each branch of $\Lambda^{i}, i=0,1$, can be extended along any continuous path avoiding the singularities of $\Lambda^{i}$.

(ii) The only singularities of $\Lambda^{i}$ over the $\tau$-plane are algebraic ramification points (of order two).

(iii) The ramification points accumulate at infinity.

Proof: A similar result was proved by F. W. Schäfke in the case of the Mathieu equations $[2,22,23,24]^{9}$. For the case of the spherical functions there is a short proof of the essential points in [2], Satz 1 p. 268 (for more details cf. [14, 25, 26, 27]).

We denote by $Z^{i}, i=0,1$, the graph of $\Lambda^{i}$ in $\mathbb{C}^{2}$.

We can prove easily $Z^{0} \cup Z^{1} \subset \mathcal{Z}$.

The preceding results suggest strongly the following conjecture (cf. [28]). A similar statement for the Mathieu equations is true, the function $F$ being replaced by the Hill determinant (cf. [24]).

Conjecture 2: (i) All the branches of the eigenvalues $\mu(\tau)$ corresponding to the even (resp. odd) eigenfunctions form a ramified multi-valued analytic function whose graph $\mathcal{Z}^{0}$ (resp. $\mathcal{Z}^{1}$ ) is an analytic curve of $\mathbb{C}^{2}$, a connected component of $\mathcal{Z}$.

(ii) We have $\mathcal{Z}=\mathcal{Z}^{0} \cup \mathcal{Z}^{1}$ and $\mathcal{Z}^{0} \cap \mathcal{Z}^{1}=\varnothing$. The curves $\mathcal{Z}^{0}$ and $\mathcal{Z}^{1}$ are the connected components of the curve $\mathcal{Z}$.

\footnotetext{
${ }^{9}$ In this case the graph is connected.
} 
(iii) The only singularities of $\mathcal{Z}$ over the $\tau$-plane, for the projection $(\tau, \mu) \mapsto \tau$, are algebraic ramification points (of order two).

(iv) For every bounded set $K$ in the $\tau$-plane, there are only finitely many ramification points of the two multivalued functions over $K$.

(v) We have $Z^{i}=\mathcal{Z}^{i}, i=0,1$.

For the proof of the conjecture the delicate point is (iv). If this statement is true, then (v) follows easily (any germ of regular branch of $\mu$ at $\tau=\tau_{0} \in \mathbb{C}$ can be extended analytically along a continuous path ending at $\tau=0$ ). Then the other statements follows from the Theorem 6. It is perhaps possible to prove (iii) using the methods of Volkmer [24].

Conjecture 3: For all $n \in \mathbb{N}$ we denote $V\left(P_{2 n}\right):=\left\{(\tau, \mu) \in \mathbb{C}^{2} \mid P_{2 n}(\tau, \mu)=0\right\}$. Then the current of integration ${ }^{10}$ on the algebraic curve $V\left(P_{2 n}\right)$ tends, when $n$ tends to infinity, to the current of integration on the analytic curve $\mathcal{Z}^{0}$.

There are similar conjectures (mutatis mutandis) for the polynomials $Q_{2 n+1}, U_{n}, \tau^{n} V_{n}$ and $D_{n}$.

Remark It would be interesting to find the order (cf. [28]) of the current of integration on the analytic curve $\mathcal{Z}^{0}$ and to study the growth of the entire function $F$.

Results similar to some results and to the conjecture ${ }^{11}$ above were recently proved by Eremenko and Gabrielov for the anharmonic oscillator and other cases [19, 20] (cf. part $10)^{12}$. But it seems that in such situations there exists nothing similar to the phenomena described in the conjecture 3 .

\section{Graphical illustration}

In this short section, we include the first graphical plots, which give us the idea of the result proved in this paper. For the two figures, the value of $\tau$ is fixed to 1 .

On the left, we plot the graphs of $V_{9}(\mu)$ (in red), $V_{10}$ (in blue) and $V_{11}$ (in green) for $\mu$ varying from 0 to 30 . We see that the polynomials cancel near the first eigenvalues of $\mathcal{D}_{1}$ [15]: $0.319,2.593,6.533,12.514,20.508$.

On the right, we plot the graphs of $V_{19}$ (in green), $V_{20}$ (in blue), and $V_{21}$ (in brown) in the neighborhood of the first eigenvalue 0.319000 .

\footnotetext{
${ }^{10} \mathrm{Cf} .[29,28,30]$.

${ }^{11}$ In the work of Eremenko and Gabrielov the analog of our conjecture is a theorem.

${ }^{12}$ Such results could be related to a Galois theory.
} 

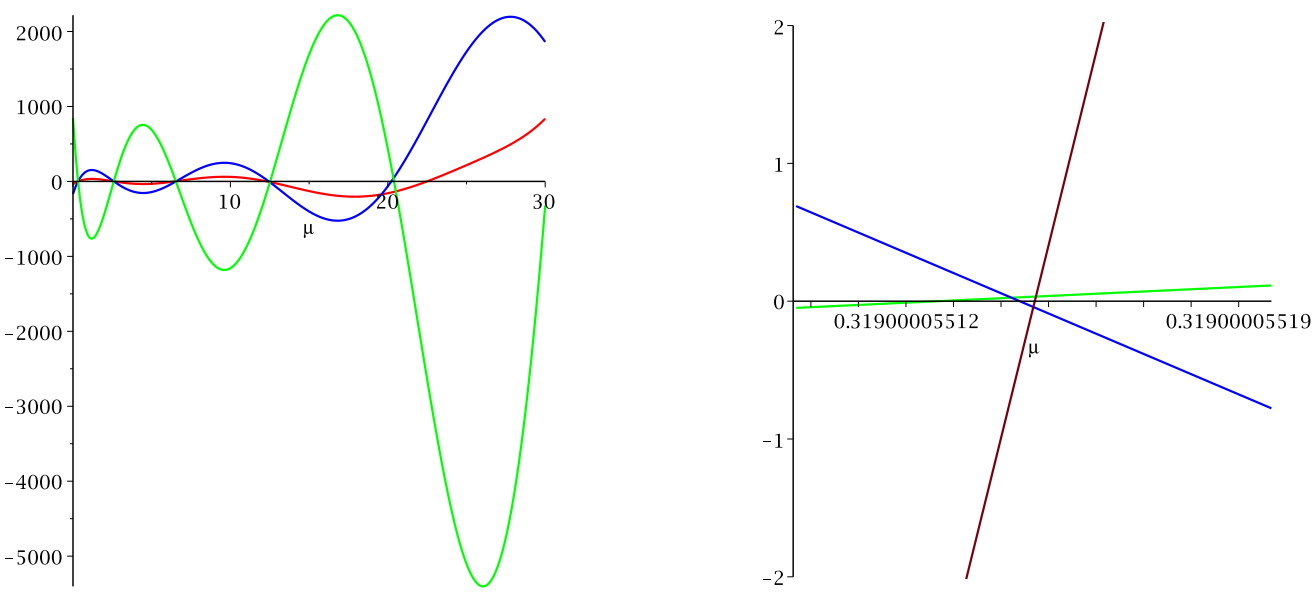

\section{Conclusion}

After many numerical experiments (cf. Appendix) we found a strong evidence in favour of the following conjecture and its variations. (We denote $\delta_{\eta}$ the Dirac mass at $\eta \in \mathbb{C} \approx \mathbb{R}^{2}$.)

Conjecture 4: For $\tau \geq 0$, we denote by $\left\{\mu_{r}(\tau)\right\}_{r \in \mathbb{N}}$ the set of eigenvalues of $\mathcal{D}_{\tau}$ and, for all $n \in \mathbb{N}$, by $\left\{\nu_{p}(\tau)\right\}_{p=0, \ldots, n-1}$ the set of roots of the polynomial $P_{2 n}{ }^{13}$ (as a polynomial in $\mu, \tau$ being fixed).

For all $\tau \geq 0$ fixed, the measure $\sum_{p=0, \ldots, n-1} \delta_{\nu_{p}(\tau)}$ (on $\mathbb{C} \approx \mathbb{R}^{2}$ ) tends to the measure $\sum_{p \in \mathbb{N}} \delta_{\mu_{2 p}(\tau)}$ (interpreted as a measure on $\mathbb{C} \approx \mathbb{R}^{2}$ ) when $n$ tends to $+\infty$.

There are similar conjectures (mutatis mutandis) replacing the sequence of polynomials $P_{2 n}$ by the sequences $Q_{2 n+1}, U_{n}$ and $\tau^{n} V_{n}$.

We proved the conjecture and its variants for "small values" of $\tau$ (cf. corollary 3 ). We explained in part 10 why the idea to prove such results for "big values" of $\tau$ replacing expansions into Legendre polynomials by expansions into parabolic cylinder functions does not work "naively".

In [5] the similar conjecture is proved for the polynomials $D_{n}$.

The above conjecture $4^{14}$ follows easily from the conjecture 3 . As the conjecture 3 seems difficult to prove, it is perhaps possible to prove directly the conjecture 4 using the proposition 2 and the analytic continuation of the eigenvalues (cf. the theorem 6). The technics of [14] could be useful.

If we admit the conjecture 3 , then we can prove that the union of the finite ramification sets of the algebraic varieties $V\left(P_{2 n}\right)$ and $V\left(Q_{2 n+1}\right)$ (and similarly the ramification sets of the algebraic varieties $\left.V\left(U_{n}\right), V\left(\tau^{n} V_{n}\right), V\left(D_{n}\right)\right)$, for the projection $(\tau, \mu) \mapsto \tau$, admits as

\footnotetext{
${ }^{13}$ The roots are complex numbers and if necessary they are repeated according to their multiplicity.

${ }^{14}$ And its generalizations for $\tau \in \mathbb{C}$.
} 
"limit" the ramification set of $\mathcal{Z}$, for the same projection ${ }^{15}$, when $n$ tends to $+\infty$. It would be interesting to try to prove this statement directly and also to test it numerically. (About such phenomena cf. [4], 7.5, in particular Possibility 1, page 354).

For sake of simplicity in this paper and our preceding work [1] we worked with prolate differential equations of order 0 . It seems easy to extend the results (and the conjectures) to the prolate and oblate differential equations of arbitrary order $m \in \mathbb{N}^{+}$:

$$
\begin{aligned}
& \left(x^{2}-1\right) y^{\prime \prime}+2 x y^{\prime}+\left(\tau^{2} x^{2}-\frac{m^{2}}{x^{2}-1}\right) y=\mu y, \\
& \left(x^{2}-1\right) y^{\prime \prime}+2 x y^{\prime}-\left(\tau^{2} x^{2}+\frac{m^{2}}{x^{2}-1}\right) y=\mu y .
\end{aligned}
$$

It would also be interesting to consider the case of the Mathieu differential equation and more generally the case of general confluent Heun equations.

\section{Aknowledgements}

Jean-Pierre Ramis thanks Bernard Malgrange who draw his attention to the work of A. Eremenko and A. Gabrielov [19] and suggested a possible relation with a Galois theory.

\footnotetext{
${ }^{15}$ That is $\left\{\tau \in \mathbb{C} \mid \frac{\partial}{\partial \mu} F(\tau, \mu)=0\right\}$
} 


\section{Appendix}

In the following array, the reader can find the first eigenvalues of $\mathcal{D}_{10}$. The first column contains the eigenvalues computed in [31], the second column contains the roots of the associated polynomial $P_{80}$ (which is a polynomial of degree 40 and has 32 real roots), the third one the roots of $Q_{81}$ (which is also of degree 40 and has 32 real roots), the fourth one the roots of $U_{80}$ or the roots of $V_{80}$ (which both are polynomials of degree 80 and have the same 60 real roots).

\begin{tabular}{|c|c|c|c|c|c|c|c|c|c|}
\hline & [31] & $P_{80}$ & $Q_{81}$ & $U_{80}$ or $V_{80}$ & & [31] & $P_{80}$ & $Q_{81}$ & $U_{80}$ or $V_{80}$ \\
\hline 0 & 9.2283043 & 9.228304297 & & 9.228304297 & & & & & \\
\hline 1 & 28.133464 & & 28.13346373 & 28.13346373 & 21 & & & 512.7076800 & 512.7076837 \\
\hline 2 & 45.868953 & 45.86895265 & & 45.86895265 & 22 & & 556.6457413 & & 556.6457564 \\
\hline 3 & 62.257700 & & 62.25770045 & 62.25770045 & 23 & & & 602.5916176 & 602.5915522 \\
\hline 4 & 76.993289 & 76.99328882 & & 76.99328882 & 24 & & 650.5440426 & & 650.5438110 \\
\hline 5 & 89.739267 & & 89.73926724 & 89.73926724 & 25 & 700.50200 & & 700.5019976 & 700.5031632 \\
\hline 6 & 101.03543 & 101.0354307 & & 101.0354307 & 26 & & 752.4646553 & & 752.4681217 \\
\hline 7 & 112.88107 & & 112.8810658 & 112.8810658 & 27 & & & 806.4313378 & 806.4108043 \\
\hline 8 & 127.05083 & 127.0508253 & & 127.0508253 & 28 & & 862.4014856 & & 862.3522979 \\
\hline 9 & 143.87201 & & 143.8720080 & 143.8720080 & 29 & & & 920.3746333 & 920.7284673 \\
\hline 10 & 163.09665 & 163.0966527 & & 163.0966527 & 30 & 980.35039 & 980.3503914 & & 981.0345614 \\
\hline 11 & 184.54762 & & 184.5476186 & 184.5476186 & 31 & & & 1042.328432 & 1036.867273 \\
\hline 12 & 208.13839 & 208.1383893 & & 208.1383893 & 32 & & 1106.308476 & & 1101.726081 \\
\hline 13 & 233.82295 & & 233.8229509 & 233.8229509 & 33 & & & 1172.290287 & 1229.956151 \\
\hline 14 & 261.57378 & 261.5737819 & & 261.5737819 & 34 & & 1240.273662 & & 1379.347766 \\
\hline 15 & 291.37313 & & 291.3731261 & 291.3731261 & 35 & 1310.2584 & & 1310.258427 & 1536.553711 \\
\hline 16 & 323.20895 & 323.2089505 & & 323.2089505 & 36 & & 1382.244431 & & 1703.373951 \\
\hline 17 & 357.07281 & & 357.0728053 & 357.0728053 & 37 & & & 1456.231543 & 1880.007184 \\
\hline 18 & 392.95859 & 392.9585889 & & 392.9585890 & 38 & & 1532.219648 & & 2066.700416 \\
\hline 19 & 430.86179 & & 430.8617935 & 430.8617933 & 39 & & & 1610.208648 & 2263.751344 \\
\hline 20 & 470.77902 & 470.7790239 & & 470.7790229 & 40 & 1690.1985 & 1690.198455 & & 2471.510319 \\
\hline
\end{tabular}




\section{References}

1. F. Fauvet, J.-P. Ramis, F. Richard-Jung, J. Thomann, Stokes phenomenon for the prolate wave equation, Applied Numerical Mathematics, Elsevier, 60 (12), pp.1309-1319, (2010). $<10.1016 /$ j.apnum.2010.05.010 $>$.

2. J. Meixner and F. W. Schäfke, Mathieusche funktionen und Sphäroidfunktionen, Springer-Verlag, (1954).

3. C. Flammer, Spheroidal Wave Functions, Stanford University Press (1975).

4. C. M. Bender, S. A. Orszac, Advanced Mathematical Methods for Scientists and Engineers, International Student Edition, McGraw-Hill International Book Company.

5. Y. Miyazaki, N. Asai, D. Cai, Y. Ikebe,, Numerical Computation of the Eigenvalues for the Spheroidal Wave Equation with Accurate Error Estimation by Matrix Method, Electronic Transactions on Numerical Analysis. Vol. 23, 329-338, (2006).

6. J. Della Dora, C. Di Crescenzo and E. Tournier, An algorithm to obtain formal solutions of a linear homogeneous differential equation at an irregular singular point, in EUROSAM 82, ed. J. Calmet, volume 144 of Lecture Notes in Computer Science page 273. Springer-Verlag, Berlin and Heidelberg (1982).

7. E. Pflügel, On the latest version of DESIR-II, Theor. Comput. Sci. 187 (1-2), 81- 86 (1997).

8. F. Richard-Jung, The DESIR Package, Software demonstration, ISSAC'09, Seoul, see also http://wwwljk.imag.fr/CASYS/LOGICIELS/desir2009.html (2009).

9. M. A. BARkAtou, Rational Newton algorithm for computing formal solutions of linear differential equations, in Proceedings of ISSAC'88, Rome, Italy, 183-195. ACM Press (1988).

10. T. Kato, Perturbation Theory for Linear Operators, Springer-Verlag, Berlin Heidelberg New York (1980).

11. K. O. Geddes, S. R. Czapor, G. Labahn, Algorithms for Computer Algebra, Kluwer Academic Publishers.

12. A. Connes, M. Marcolli, Noncommutative Geometry, Quantum Fields and Motives, A.M.S. Colloquium Publications, vol. 55 (2007).

13. A. Hautot, Accélération de la convergence en analyse numérique, 1ère partie : Théorie des récurrences, http://www.physinfo.org/Acc_Conv/Acc_Conv_Part1.pdf.

14. B. E. Barrowes, K. O’Neill, T. M. Grzegorczyk, Jin A. Kong, On the Asymptotic Expansion of the Spheroidal Wave Function and its Eigenvalues for Complex Size Parameter, Studies in Applied Mathematics, 113(3):271 - 301 (2003).

15. M. Abramowitz, I. A. Szegun, Pocketbook of Mathematical Functions, Verlag Harri Deutsch - Thun Frankfurt/Main (1984).

16. C. M. Bender, T. Tsun Wu, Anharmonic oscillator, Phys. Rev. (2) 184: 1231-1260 (1969).

17. E. Delabaere, F. Pham, Unfolding the Quartic Oscillator, Annals of Physics 261, 180-218 (1987).

18. G. BAŞAR, G. V. Dunne, Resurgence and the Nekrasov-Shatashvili limit: connecting weak and strong coupling in the Mathieu and Lamé systems, JHEP published for SISSA by Springer (2015).

19. A. Eremenko, A. Gabrielov, Analytic continuation of egienvalues of a quartic oscillator, Comm. Math. Phys. 287 (2), 43-457 (2009).

20. A. Eremenko, A. Gabrielov, Irreducibility of some spectral determinants, arXiv:0904.1714, (2013).

21. Y. SiBuYA, Global theory of a second order linear ordinary differential equation with a polynomial coefficient, North Holland, Amsterdam (1975).

22. J. Meixner, F. W. Schäfke, Gerhard Wolf, Mathieu Functions and Spheroidal Functions and Their Mathematical Foundations, Lecture Notes in Math., Vol. 837. Springer-Verlag, New York (1980).

23. P. N. Shivakumar, J. Xueb, On the double points of a Mathieu equation, Journal of Computational and Applied Mathematics 107: 111-125 (1999).

24. H. Volkmer, On Riemann Surfaces of Analytic Eigenvalue Functions, Complex Variables, Vol. 49 (3), 169-182 (2004).

25. B. Guerrieri, C. Hunter, The eigenvalues of the angular spheroidal wave-equation, Studies in Applied Mathematics, vol. 66 (3), 217-240 (1982). 
26. S. L. Skorokhodov, D. V. Khristoforov, Calculation of the branch points of the eigenfunctions corresponding to wave spheroidal functions, Computational Mathematics and Mathematical Physics Vol. 46 (7), 1132-1146 (2006).

27. T. OGuchi, Eigenvalues of spheroidal wave functions and their branch points for complex values of propagation constants, Radio Science, vol. 5 (8-9), 1207-14 (1970).

28. H. SkodA, Sous-ensembles analytiques d'ordre fini ou infini dans $\mathbb{C}^{n}$, Bull. Soc. Math. France, 100: 353408 (1972).

29. P. Lelong, Intégration sur un ensemble analytique complexe, Bull. Soc. Math. France, 85: 239-262 (1957).

30. G. De Rham, Differentiable manifolds, Springer (1984). (Translated from French, french version 1973).

31. D. Slepian and E. Sonnenblick, Eigenvalues associated with prolate spheroidal equation of zero order, Bell Syst. Tech. J., vol. 44, pp. 1745-1760 (1965).

Université Grenoble Alpes, LJK, UMr CNRS 5224, 41 Rue des mathúatiques, 38041 Grenoble Cedex, France

Institut de France (Académie des Sciences) and Université Paul Sabatier (Toulouse 3), IMT, UMR CNRS 5219, 118 route de Narbonne, 31062 Toulouse Cedex 4, France

Université DE Strasbourg, IRMA, UMR CNRS 7501, 7 rue René Descartes, 67084 Strasbourg Cedex, France 